\title{
514.
}

\section{ON THE PROBLEM OF THE IN-AND-CIRCUMSCRIBED TRIANGLE.}

[From the Philosophical Transactions of the Royal Society of London, vol. CLXI. (for the year 1871), pp. 369-412. Received December 30, 1870,-Read February 9, 1871.]

THE problem of the In-and-Circumscribed Triangle is a particular case of that of the In-and-Circumscribed Polygon: the last-mentioned problem may be thus stated-to find a polygon such that the angles are situate in and the sides touch a given curve or curves. And we may in the first instance inquire as to the number of such polygons. In the case where the curves containing the angles and touched by the sides respectively are all of them distinct curves, the number of polygons is obtained very easily and has a simple expression: it is equal to twice the product of the orders of the curves containing the several angles respectively into the product of the classes of the curves touched by the several sides respectively; or, say, it is equal to twice the product of the orders of the angle-curves into the product of the classes of the side-curves. But when several of the curves become one and the same curve, and in particular when the angles are all of them situate in and the sides all touch one and the same curve, it is a much more difficult problem to find the number of polygons. The solution of this problem when the polygon is a triangle, and for all the different relations of identity between the different curves, is the object of the present memoir, which is accordingly entitled "On the Problem of the In-and-Circumscribed Triangle;" the methods and principles, however, are applicable to the case of a polygon of any number of sides, the method chiefly made use of being that furnished by the theory of correspondence, as will be explained. The results (for the triangle) are given in the following Table; for the explanation of which I remark that the triangle is taken to be $a B c D e F$; viz. $a, c, e$ are the angles, $B, D, F$ the sides; that is, $B, D, F$ are the sides $a c, c e, e a$ respectively, and $a, c, e$ are the angles $F B, B D, D F$ 
respectively. And I use the same letters $a, c, e, B, D, F$ to denote the curves containing the angles and touched by the sides respectively; viz. the angle $a$ is situate in the curve $a$, the side $B$ touches the curve $B$, and so for the other angles and sides respectively. An equation such as $a=c$ or $a=B$ denotes that the curves $a, c$ or, as the case may be, the curves $a, B$ are one and the same curve: it is in general convenient to use a new letter for denoting these identical curves; viz. I write, for instance, $a=c=x$ or $a=B=x$, to denote that the curves $a, c$ or, as the case may be, the curves $a, B$ are one and the same curve $x$; the new letters thus introduced are $x, y, z$, there being in regard to them no distinction of small letters and capitals. The expression "no identities" denotes that the curves are all distinct. But I use also the letters $a, c, e, b, d, f, x, y, z$, and $A, C, E, B, D, F, X, Y, Z$ quantitatively, to denote the orders and classes of the curves $a, c, e, B, D, F, x, y, z$ respectively; thus, in the Table, for the case 1 "no identities" the number of triangles is given as $=2 a c e B D F$, which agrees with the before-mentioned result for the polygon: for the case 2 the several separate identities $a=c, a=e, c=e$ are of course equivalent to each other; and selecting one of them, $a=c=x$, the number of triangles is given as $=2 x(x-1) e B D F$. There is a convenience in thus writing down the several forms $a=c, a=e, c=e$ of the identity or identities which constitute the 52 distinct cases of the Table; and I have accordingly done so throughout the Table, the expression for the number of triangles being however in each case given under one form only. It only remains to mention that for the curve $x$ the Greek letter $\xi$ denotes what may be termed the "stativity" of the curve, viz. this is = number of cusps +3 times the class, or, what is the same thing, = number of inflections +3 times the order; the curve being determined by its order $x$, class $X$, and $\xi$; and similarly for $\eta$ and $\zeta$.

Observe that, in the column "Specification," each line is to be read separately from the others, and, where the word "or" occurs, the two parts of the line are to be read separately; thus case 5 , the six forms are $a=B, a=F, c=D, c=B, e=F, e=D$ : the letter $x$ (or, as the case may be, $x, y$, or $x, y, z$ ) accompanies the first of the given forms; in the present instance $a=B=x$, and it is to this first form that the number of triangles, here $2(X x-X-x) c e D F$, applies.

I remark that what is primarily determined is the number of positions of a particular angle of the triangle, and that in some cases, on account of the symmetry of the figure, the number of triangles is a submultiple of this number; viz. the number of positions of the angle is to be divided by 2 or 6 ; this is expressly shown, by means of a separate column, in the Table. 


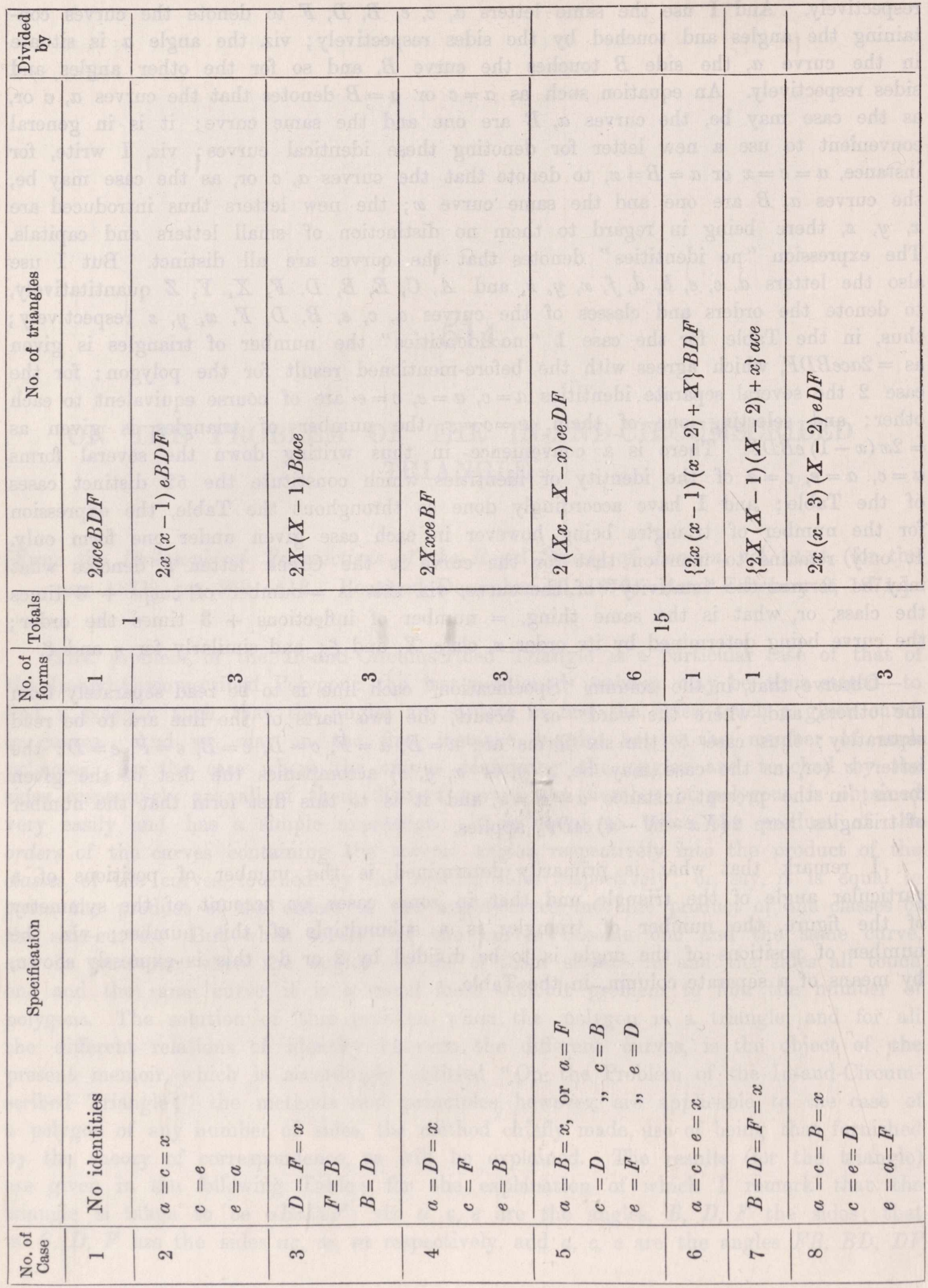




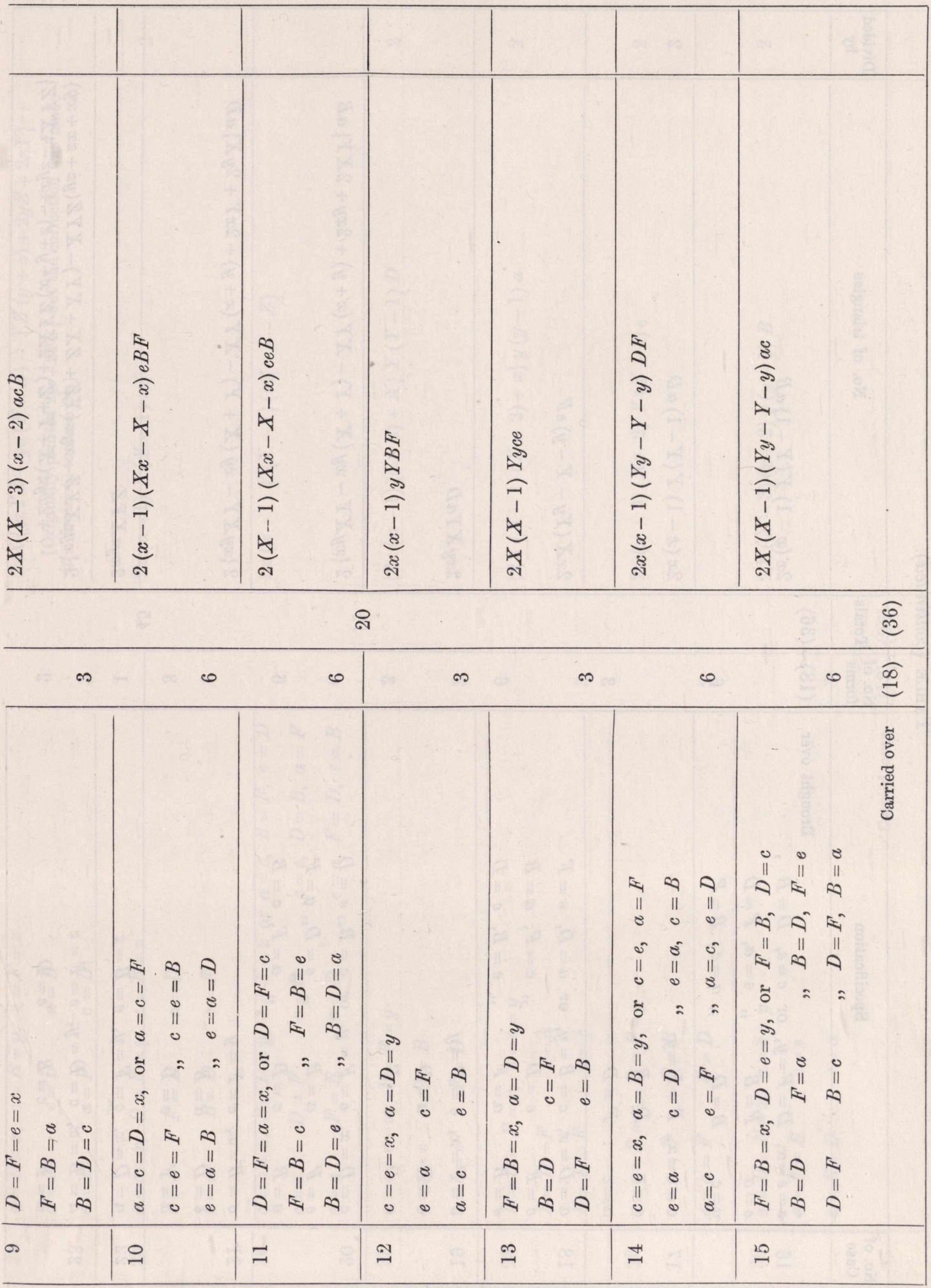




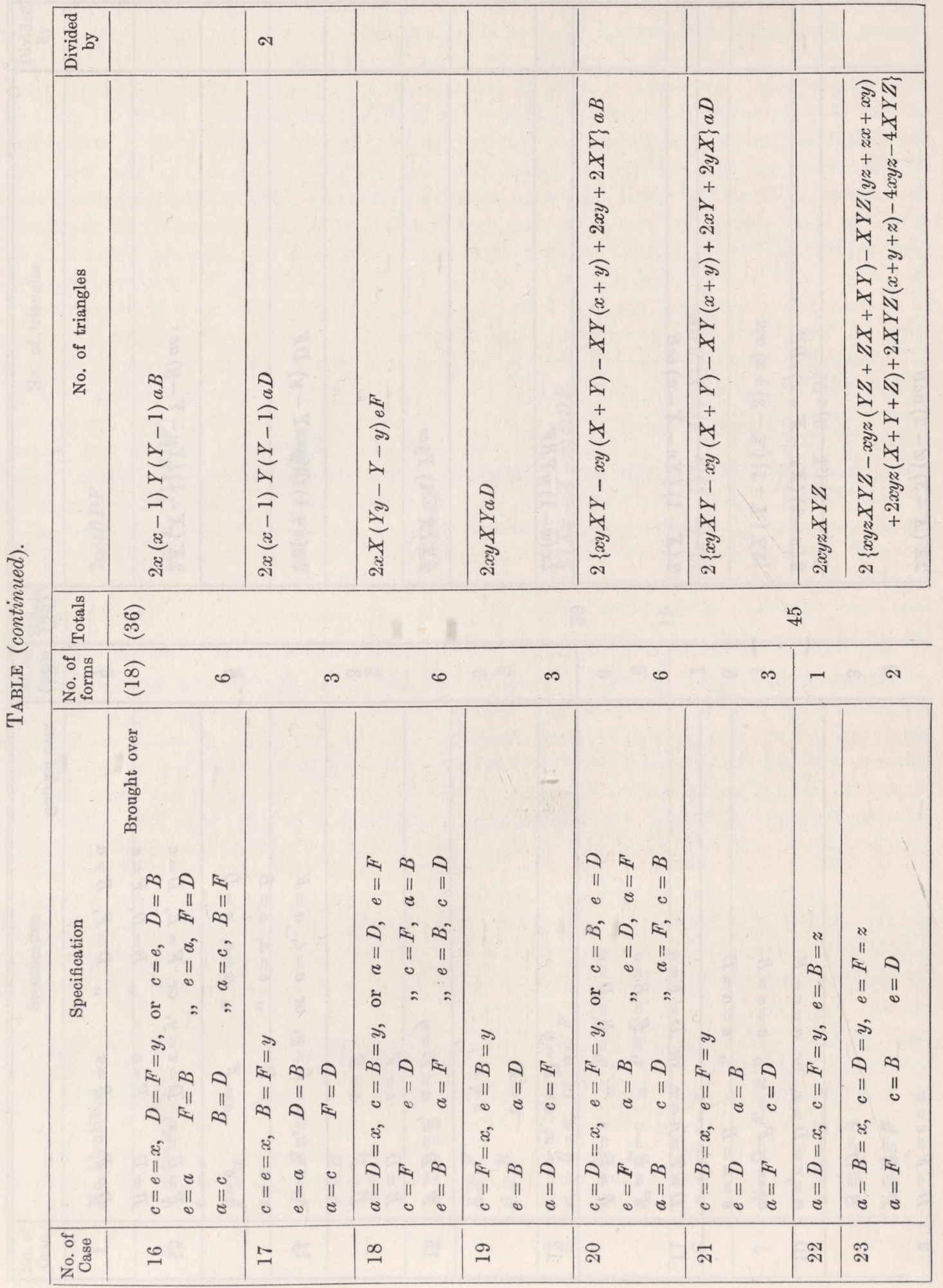




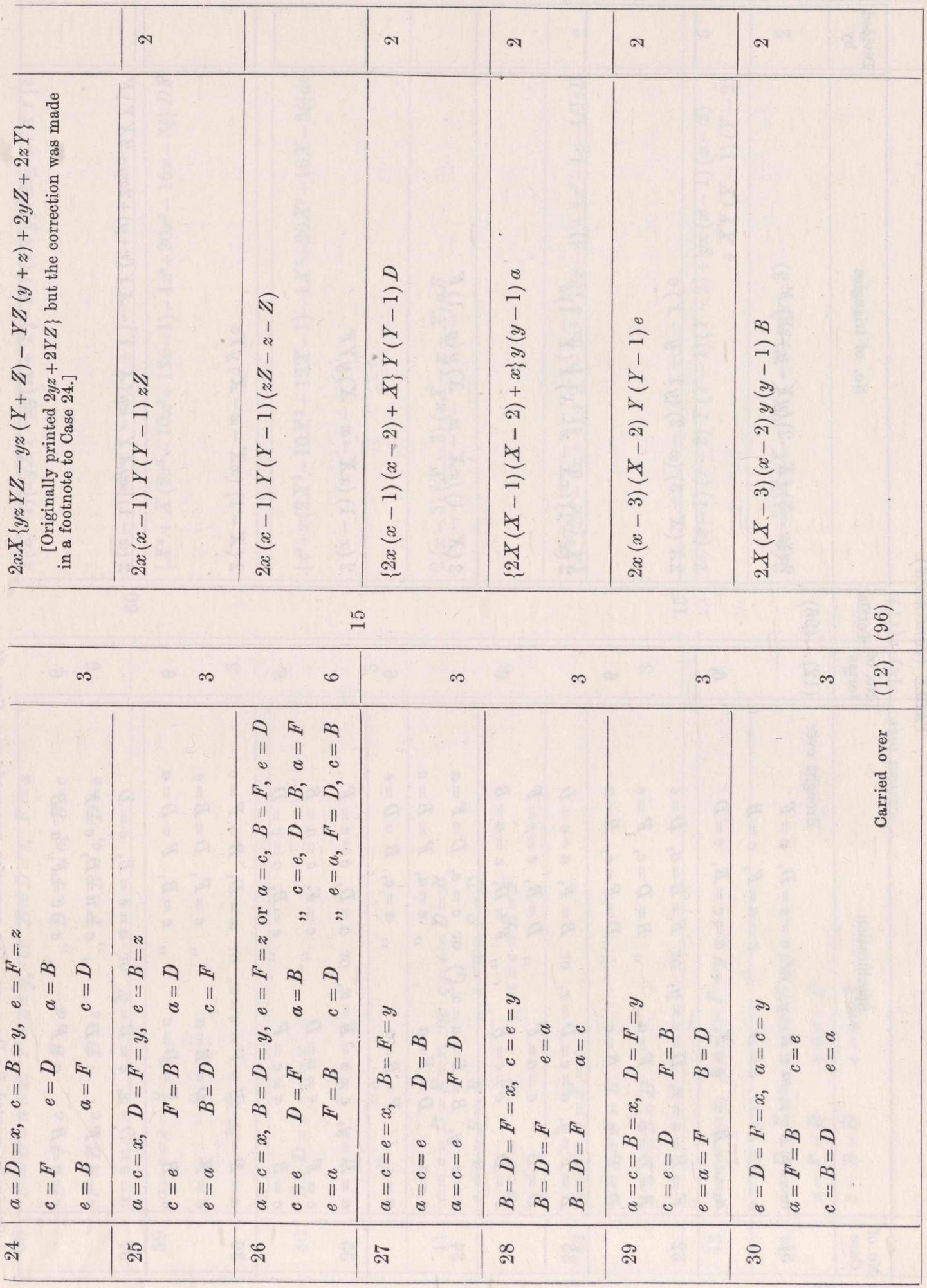




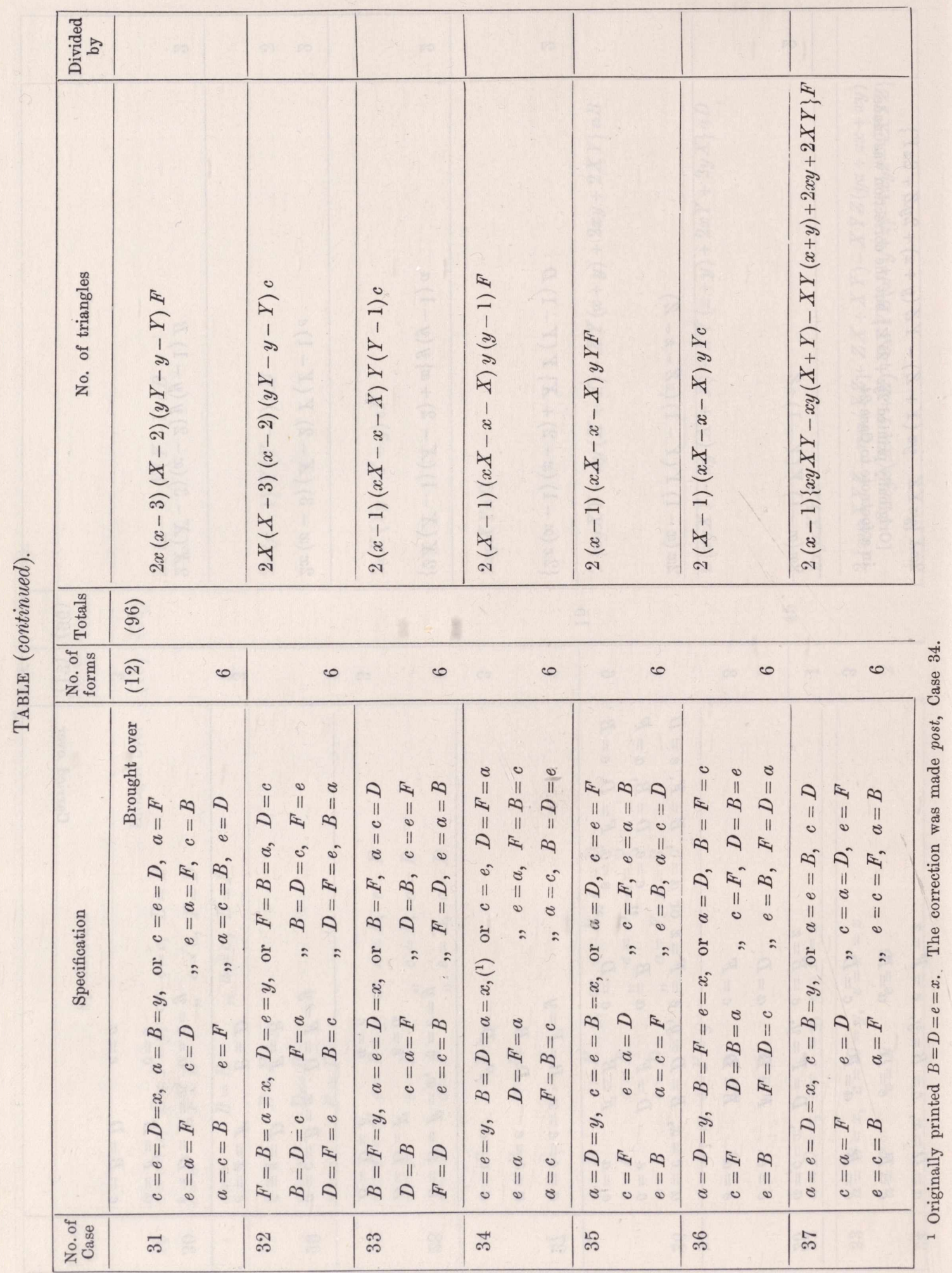




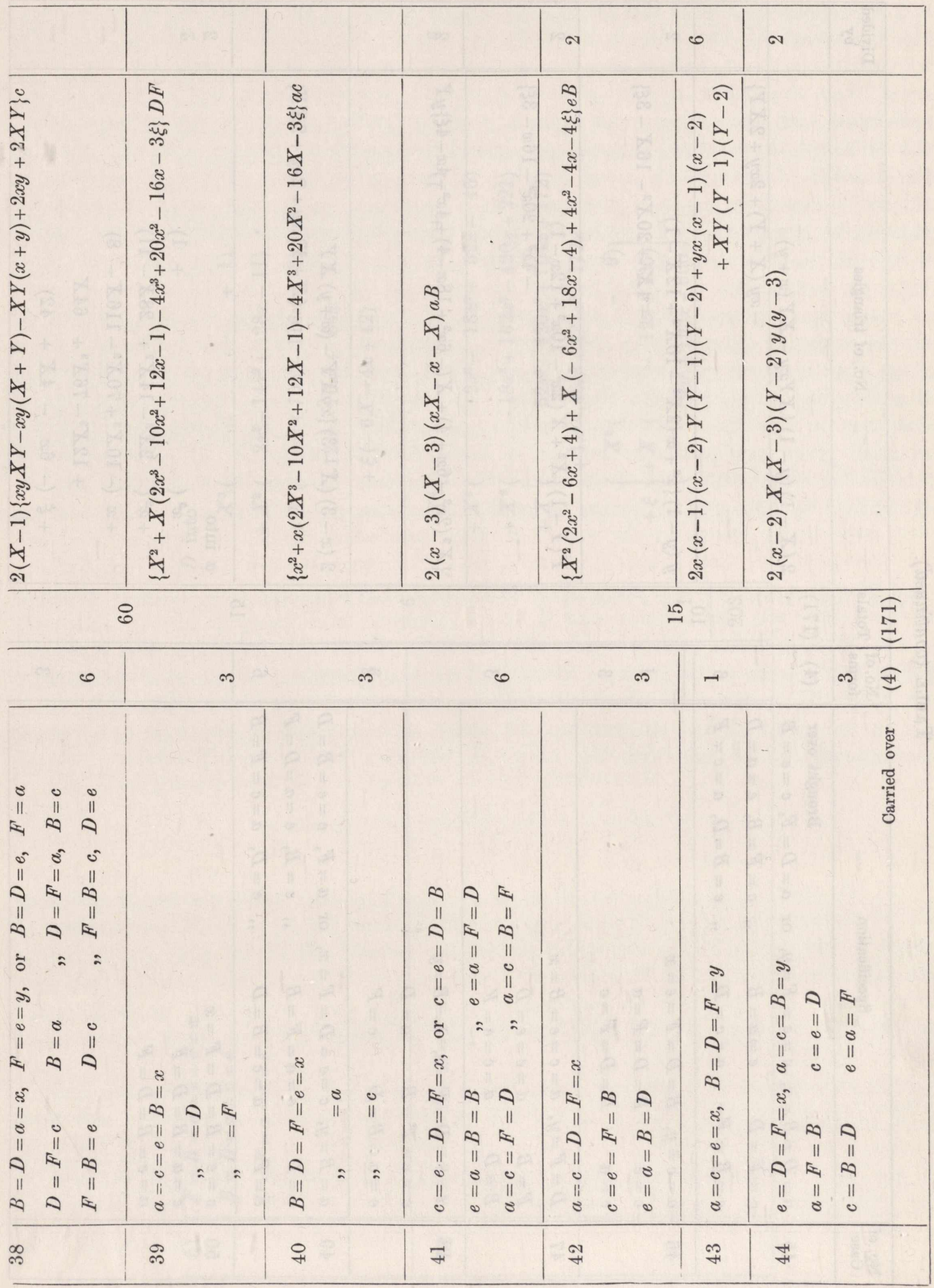




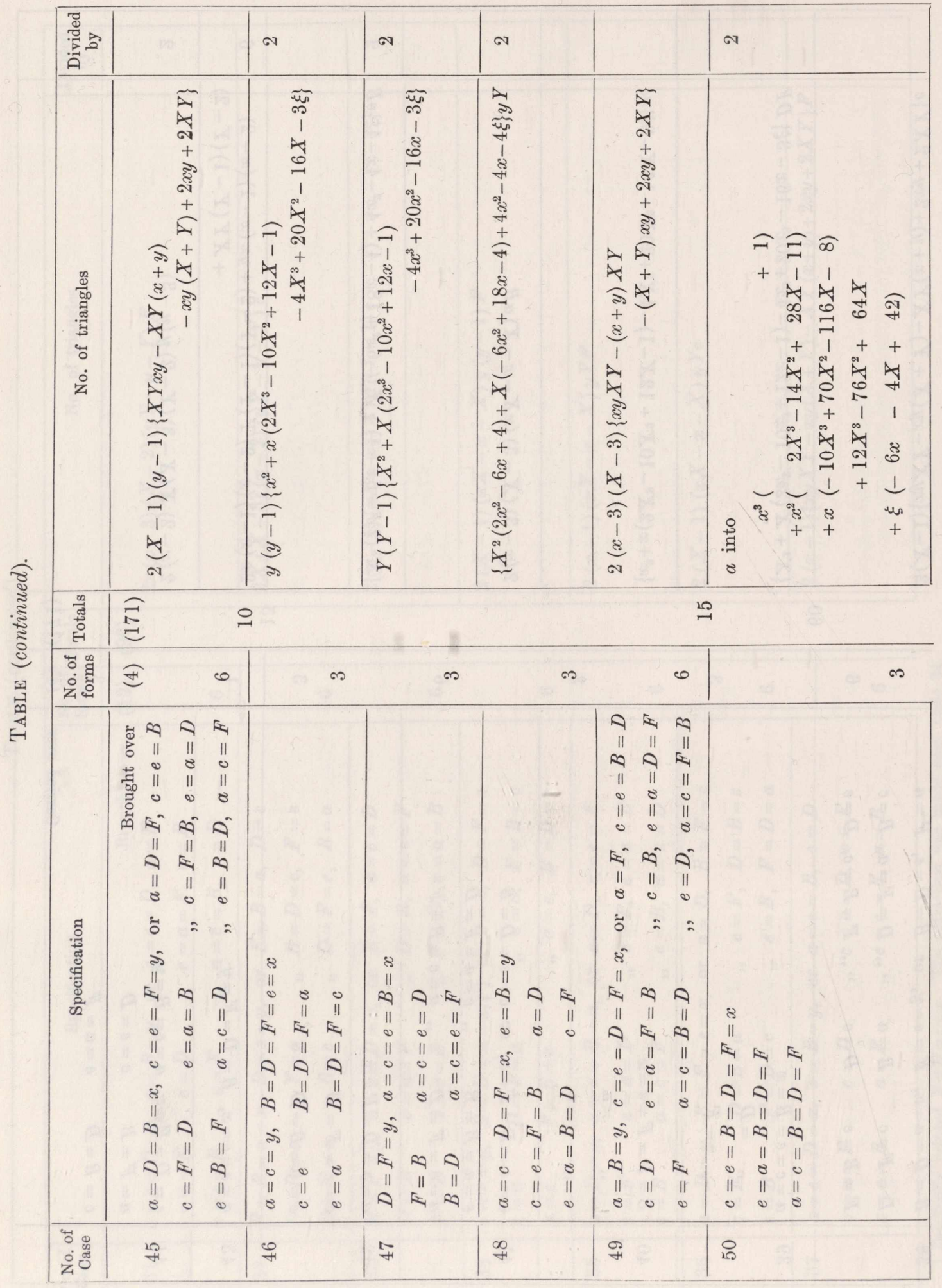




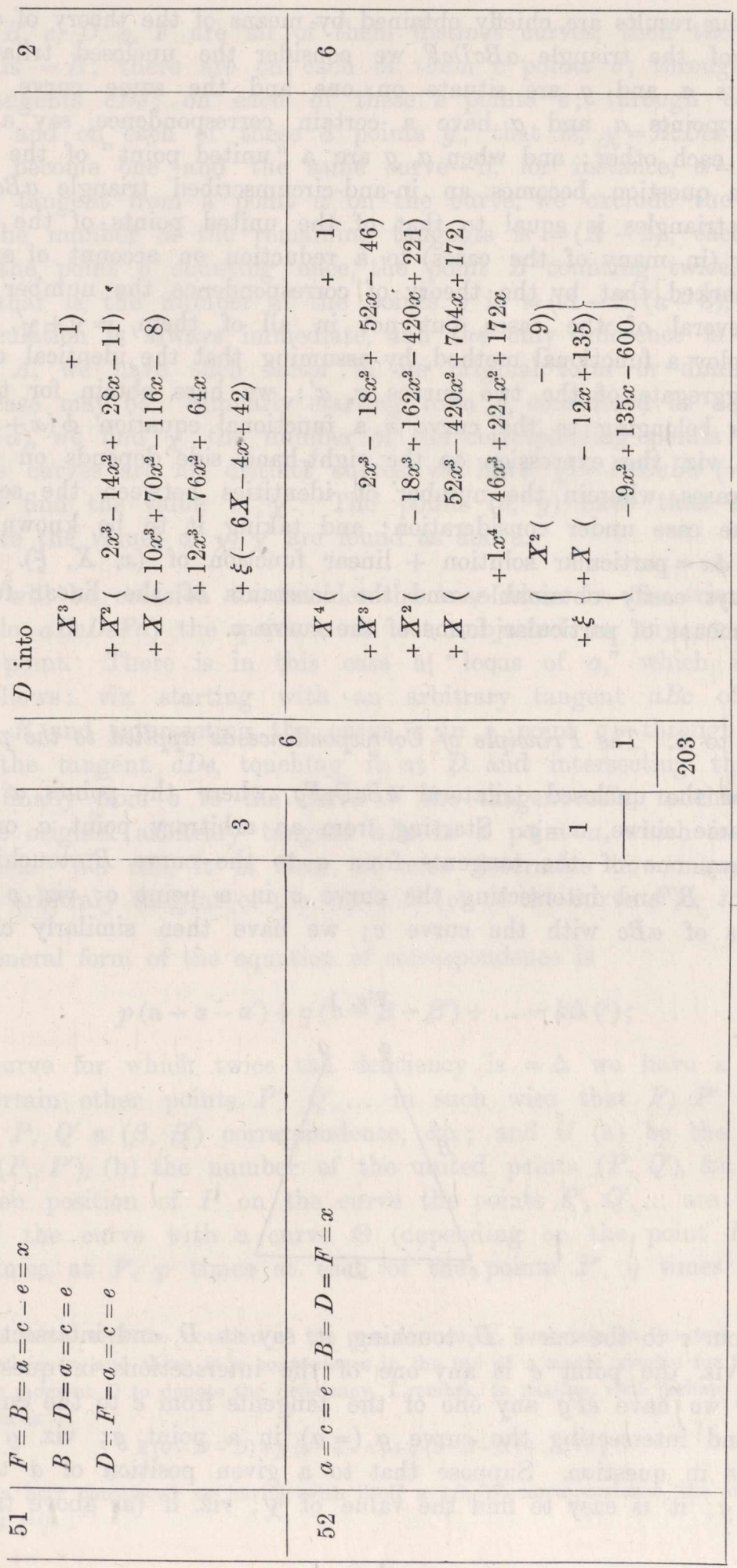


The foregoing results are chiefly obtained by means of the theory of correspondence; viz. if instead of the triangle $a B c D e F$ we consider the unclosed trilateral $a B c D e F g$, where the points $a$ and $g$ are situate on one and the same curve, say the curve $a=g$, then the points $a$ and $g$ have a certain correspondence, say a $\left(\chi, \chi^{\prime}\right)$ correspondence with each other; and when $a, g$ are a "united point" of the correspondence, the trilateral in question becomes an in-and-circumscribed triangle $a B c D e F$; that is, the number of triangles is equal to that of the united points of the correspondence, subject however (in many of the cases) to a reduction on account of special solutions. It may be remarked that by the theory of correspondence the number of the united points is, in several of the cases, but not in all of them, $=\chi+\chi^{\prime}$. But in some instances I employ a functional method, by assuming that the identical curves are each of them the aggregate of the two curves $x, x^{\prime}$ : we here obtain for the number $\phi x$ of the triangles belonging to the curve $x$ a functional equation $\phi\left(x+x^{\prime}\right)-\phi x-\phi x^{\prime}=$ given function; viz. the expression on the right-hand side depends on the solution of the preceding cases, wherein the number of identities between the several curves is less than in the case under consideration; and taking it to be known, the functional equation gives $\phi x=$ particular solution + linear function of $(x, X, \xi)$. The particular solution is always easily obtainable, and the constants of the linear function can be determined by means of particular forms of the curve $x$.

Article Nos. 1 to 6. The Principle of Correspondence as applied to the present Problem.

1. Consider the unclosed trilateral $a B c D e F g$, where the points $a$ and $g$ are on one and the same curve, $a=g$. Starting from an arbitrary point $a$ on the curve $a$, we have $a B c$ any one of the tangents from $a$ to the curve $B$, touching this curve, say at the point $B$, and intersecting the curve $c$ in a point $c$; viz. $c$ is any one of the intersections of $a B c$ with the curve $c$; we have then similarly $c D e$ any one of

Fig. 1.

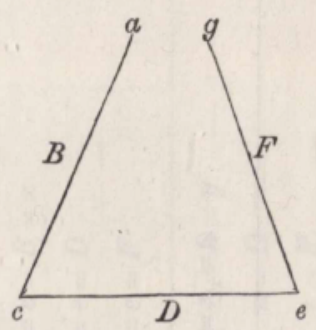

the tangents from $c$ to the curve $D$, touching it, say at $D$, and intersecting the curve $e$ in a point $e$; viz. the point $e$ is any one of the intersections in question; and then in like manner we have $e F g$ any one of the tangents from $e$ to the curve $F$, touching it, say at $F$, and intersecting the curve $g(=a)$ in a point $g$; viz. $g$ is any one of the intersections in question. Suppose that to a given position of $a$ there correspond $\chi$ positions of $g$; it is easy to find the value of $\chi$; viz. if (as above tacitly supposed) 
the curves $a, B, c, D, e, F$ are all of them distinct curves, then the number of the tangents $a B c$ is $=B$; there are on each of them $c$ points $c$; through each of these we have $D$ tangents $c D e$; on each of these $e$ points $e$; through each of these $F^{\prime}$ tangents $c F g$; and on each of these $a$ points $g$; that is, $\chi=B c D e F a$. But if some of the curves become one and the same curve-if, for instance, $a=B=c$, - the line $a B c$ is here a tangent from a point $a$ on the curve, we exclude the tangent at the point $a$, and the number of the remaining tangents is $=(A-2)$; each tangent meets the curve in the point $a$ counting once, the point $B$ counting twice, and in $(a-3)$ other points; that is, the number of the points $c$ is $=(A-2)(a-3)$, and so in other cases; the calculation is always immediate, and the only difference is that, instead of a factor $a$ or $A$, we have such factor in its original form or diminished by 1, 2, or 3 , as the case may be. Similarly starting from $g$, considered as a given point on the curve $g(=a)$, we find $\chi^{\prime}$ the number of the corresponding points $a$; thus in the case where the curves are all distinct curves, we have $\chi^{\prime}=F e D c B a(=\chi)$; and so in other cases we find the value of $\chi^{\prime}$. The points $(a, g)$ have thus a $\left(\chi, \chi^{\prime}\right)$ correspondence, where the values of $\chi, \chi^{\prime}$ are found as above.

2. There will be occasion to consider the case where in the triangle $a B c D e F$ (or say the triangle $a B c D e F a$ ) the point $a$ is not subjected to any condition whatever, but is a free point. There is in this case a "locus of $a$," which is at once constructed as follows: viz. starting with an arbitrary tangent $a B c$ of the curve $B$, touching it at $B$ and intersecting the curve $c$ in a point $c$; through $c$ we draw to the curve $D$ the tangent $c D e$, touching it at $D$ and intersecting the curve $e$ in a point $e$; and finally from $e$ to the curve $F$ the tangent $e F a$, touching it at $F$ and intersecting the original arbitrary tangent $a B c$ in a point $a$, which is a point on the locus in question. We can, it is clear, at once determine kow many points of the locus lie on an arbitrary tangent of the curve $B$ (or of the curve $F$ ).

3. The general form of the equation of correspondence is

$$
p\left(\mathrm{a}-\alpha-\alpha^{\prime}\right)+q\left(\mathrm{~b}-\beta-\beta^{\prime}\right)+\ldots=k \Delta\left({ }^{1}\right) ;
$$

viz. if on a curve for which twice the deficiency is $=\Delta$ we have a point $P$ corresponding to certain other points $P^{\prime}, Q^{\prime}, \ldots$ in such wise that $P, P^{\prime}$ have an $\left(\alpha, \alpha^{\prime}\right)$ correspondence, $P, Q^{\prime}$ a $\left(\beta, \beta^{\prime}\right)$ correspondence, \&c.; and if (a) be the number of the united points $\left(P, P^{\prime}\right)$, (b) the number of the united points $\left(P, Q^{\prime}\right)$, \&c. ; and if moreover for a given position of $P$ on the curve the points $P^{\prime}, Q^{\prime}, \ldots$ are obtained as the intersections of the curve with a curve $\Theta$ (depending on the point $P$ ) which meets the curve $k$ times at $P, p$ times at each of the points $P^{\prime}, q$ times at each of the

1 To avoid confusion with the notation of the present memoir, I abstain in the text from the use of $D$ as denoting the deficiency, and there is a convenience in the use of a single symbol for twice the deficiency; but writing for the moment $D$ to denote the deficiency, I remark, in passing, that perhaps the true theoretical form of the equation is

$$
k(0-D-D)+p\left(\mathrm{a}-a-a^{\prime}\right)+q\left(\mathrm{~b}-\beta-\beta^{\prime}\right)+\ldots=0 ;
$$

viz. the point $P$ is here considered as having with itself a $(D, D)$ correspondence, the number of the united points therein being $=0$. 
points $Q$, \&c.; then the relation between the several quantities is as stated above: see my "Second Memoir on the Curves which satisfy given conditions," Philosophical Transactions, vol. 159 (1868), pp. 145-172, [407]. I omit for the present purpose the term "Supp.," treating it as included in the other terms.

4. In the present case we consider, as already mentioned, the unclosed trilateral $a B c D e F g$, where the angles $a, g$ are on one and the same curve $a(=g)$ (the curve in the general theorem); and the curve $\Theta$ is the system of lines $e F g$ which by their intersection with the curve $a$ determine the points $g$. Considering these as the points $\left(P, P^{\prime}\right)$ of the general theorem we have $p=1$ : I change the notation, and instead of a $-\alpha-\alpha^{\prime}$ write $g-\chi-\chi^{\prime}$; viz. I take (g) for the number of the united points $(a, g)$, and suppose that the points $(a, g)$ have a $\left(\chi, \chi^{\prime}\right)$ correspondence. The most simple case is when the curve $a$ is distinct from each of the curves $e, F$; here all the intersections of the line-system $e F g$ with the curve $a$ are points $g$, that is we have only the correspondence $(a, g)$; and since the line-system $e F g$ does not pass through the point $a$, we have simply

$$
g-\chi-\chi^{\prime}=0
$$

5. But suppose that the curves $a, e, F$ are one and the same curve, say that $a=e=F$; understanding by the point $F$ the point of contact of a line $e F g$ with the curve $a$, then the intersections of the line-system $e F g$ with the curve $a$ are the points $g$ each once, the points $F$ each twice, and the points $e$ each as many times as there are lines $\mathrm{eFg}$ through the point $e$, say erch $M$ times. (In the present case, where the curves $e, F$ are identical, we have $M=F-2$ or $F-3$ according as the curve $D$ is or is not distinct from the curve $F$; in the cases afterwards referred to, the values may be $F$ or $F-1$; that is, we have always $M=F, F-1, F-2, F-3$, as the case may be.) We have to consider the several correspondences $(a, g),(a, F),(a, e) ; k$ is as before $=0$; and the form of the theorem is

$$
\left(\mathrm{g}-\chi-\chi^{\prime}\right)+2\left(\mathrm{f}-\phi-\phi^{\prime}\right)+M\left(\mathrm{e}-\epsilon-\epsilon^{\prime}\right)=0,
$$

where the symbols denote as follows, viz.

$(a, g)$ have a $\left(\chi, \chi^{\prime}\right)$ correspondence, and No. of united points $=\mathrm{g}$,

$$
\begin{aligned}
& \left(a, F^{\prime}\right) \quad "\left(\phi, \phi^{\prime}\right) \quad \text { " } \quad \text { " } \\
& (a, e) \#\left(\epsilon, \epsilon^{\prime}\right) \quad \# \quad \# \quad \text { " } \quad \text {, }
\end{aligned}
$$

so that the determination of $g$ here depends upon that of $f-\phi-\phi^{\prime}$ and $e-\epsilon-\epsilon^{\prime}$.

6. The curve $a$ might however have been identical with only one of the curves $e, F$; viz. if $a=F$, but $e$ is a distinct curve, then the equation will contain the term $2\left(\mathrm{f}-\phi-\phi^{\prime}\right)$, but not the term $M\left(\mathrm{e}-\epsilon-\epsilon^{\prime}\right)$; and so if $a=e$, but $F$ is a distinct curve, then the equation will not contain $2\left(\mathrm{f}-\phi-\phi^{\prime}\right)$, but will contain $M\left(\mathrm{e}-\epsilon-\epsilon^{\prime}\right)$ : it is to be noticed that in this last case we have $M=F$ or $M=F-1$, according as the curve $D$ is not, or is, one and the same curve with $F$. The determination of $(\mathrm{g})$ here depends upon that of $\mathrm{f}-\phi-\phi^{\prime}$ or $\mathrm{e}-\epsilon-\epsilon^{\prime}$, as the case may be. These sub- 
sidiary values $f-\phi-\phi^{\prime}$ and $e-\epsilon-\epsilon^{\prime}$ are obtained by means of a more simple application of the principle of correspondence, as will appear in the sequel( $\left.{ }^{1}\right)$, but for the moment I do not pursue the question.

\section{Article Nos. 7 to 14. Locus of a free angle (a).}

7. I consider the case where $a$ is a distinct curve $\neq e, \neq F$, and where, as was seen, the equation is simply

$$
g-x-\dot{x}^{\prime}=0
$$

I suppose further that $a$ is distinct from all the other curves, or say, simpliciter, that $a$ is a distinct curve. The values of $\chi, \chi^{\prime}$ will here each of them contain the factor $a$, say we have $\chi=a \omega, \chi^{\prime}=a \omega^{\prime}$; and therefore the equation gives $\mathrm{g}=a\left(\omega+\omega^{\prime}\right)$. It is obvious that $\omega, \omega^{\prime}$ are the values assumed by $\chi, \chi^{\prime}$ respectively in the particular case where the curve $a$ is an arbitrary line $(a=1)$; and $\omega+\omega^{\prime}$ is the number of the united points on this line.

8. Suppose now that in the triangle $a B c D e F a$ the point $a$ is a free point, we have, as above-mentioned, a locus of $a$, and the united points on the arbitrary line are the intersections of the line with this locus; that is, the locus meets the arbitrary line in $\omega+\omega^{\prime}$ points; or, what is the same thing, the order of the locus is $=\omega+\omega^{\prime}$.

9. I stop for a moment to remark that in the particular case where the curve $B$ is a point $(B=1)$, then in the construction of the locus of $a$ the arbitrary tangent $a B c$ is an arbitrary line through $B$, and the construction gives on this line $\omega$ positions of the point $a$. But drawing from $B$ a tangent to the curve $F$, and thus constructing in order the points $F, e, D, c, a$, the construction shows that $B$ is an $\omega^{\prime}$-tuple point on the locus; and (by what precedes) an arbitrary line through $B$ meets the locus in $\omega$ other points; that is, in the particular case where the curve $B$ is a point, the order of the locus of $a$ is $=\omega+\omega^{\prime}$, which agrees with the foregoing result.

10. The construction for the locus of a may be presented in the following form: viz. drawing to the curve $D$ a tangent $c D e$, meeting the curves $c$, $e$ in the points $c, e$ respectively; then if from any point $c$ we draw to the curve $B$ a tangent $c B a$, and from any point $e$ to the curve $F$ a tangent $e F a$, the tangents $c B a, e F a$ intersect in a point on the required locus. Hence if in any particular case (that is for any particular position of the tangent $c D e$ ) the lines $c B a, e F a$ become one and the same line, the point $a$ will be an indeterminate point on this line; that is, the line in question will be part of the locus of $a$.

11. The case cannot in general arise so long as the curves $B, F$ are distinct from each other; but when these are one and the same curve, say when $B=F$, it will arise, and that in two distinct ways. To show how this is, suppose, to fix the ideas, that the curves $c, D, e$ are distinct from each other and from the curve $B=F$. Then the first mode is that shown in the annexed "first-mode figure," viz. we have 
here a tangent at $D$ passing through a point $c e$ of the intersection of the curves $c, e$, and from this point a tangent drawn to the curve $B=F$. For the position in question of the tangent of $D$, the points $c, e$ coincide with each other, and we have thus the coincident tangents $c B a$ and $e F a$ to the identical curves $B=F$. It is further

Fig. 2. First-mode figure.

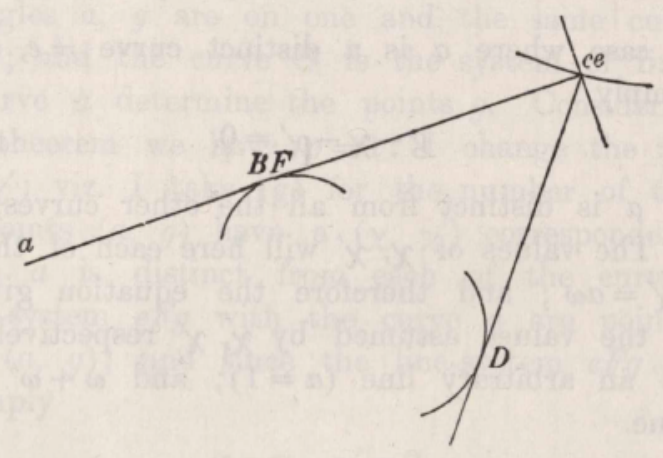

to be remarked that the number of the points of intersection is $=c e$; from each of these there are $B$ tangents to the curve $B=F$ (in all ce. $B$ tangents), and each of these counts once in respect of each of the $D$ tangents to the curve $D$, that is, it counts $D$ times. We have thus, as part of the locus of $a, c e . B$ lines each $D$ times, or, say, first-mode reduction $=c e . B . D$.

12. The second mode is that shown in the annexed "second-mode figure." The tangent from $D$ is here a common tangent of the curves $D$, and $B=F$. This meets the curve $c$ in $c$ points, and the curve $e$ in $e$ points; and attending to any pair of points $c, e$, these give the tangents $c B a, e F a$, coinciding with the common tangent in

Fig. 3. Second-mode figure.

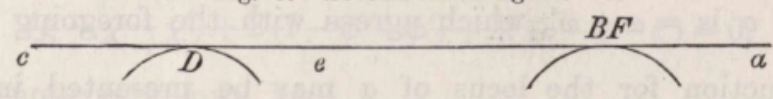

question, and forming part of the locus of $a$. The number of the common tangents is $=B D$; but each of these counts once in respect of each combination of the points $c, e$, that is in all ce times. And we have thus as part of the locus $B D$ lines each c.e times, or, say, second-mode reduction $=B D . c . e$. This is (as it happens) the same number as for the first mode; but to distinguish the different origins I have written as above ce. $B . D$ and $B D . c . e$ respectively.

13. It is important to remark that each of the two modes arises whatever relations of identity subsist between the curves $c, e, D$, and $B=F$, but with considerable modification of form. Thus if the curves $c, e$ are identical $(c=e)$ but distinct from $D$, then in the first-mode figure ce may be a node or a cusp of the curve $c=e$, or it may be a point of contact of a common tangent of the curves $D$, and $c=e$. As regards the node, remark that if we consider a tangent of $D$ meeting the curve $c=e$ in the neighbourhood of the node, then of the two points of intersection each 
in succession may be taken for the point $c$, and the other of them will be the point $e$; so that the node counts twice. It requires more consideration to perceive, but it will be readily accepted that the cusp counts three times. Hence if for the curve $c=e$ the number of nodes be $=\delta$ and that of cusps $=\kappa$, the value of the first-mode reduction is $=(2 \delta+3 \kappa+C) B D$, or, what is the same thing, it is $=\left(c^{2}-c\right) B D$.

As regards the second-mode figure, the only difference is that $c, e$ will be here any pair of intersections (each pair twice) of the tangent with the curve $c=e$; the value is thus $=\left(c^{2}-c\right) B D$.

It would be by no means uninteresting to enumerate the different cases, and indeed there might be a propriety in doing so here; but I have (instead of this) considered the several cases, when and as they arise in connexion with any of the cases of the in-and-circumscribed triangle.

14. Observe that the general result is, that in the case $B=F$ of the identity of the curves $B$ and $F$, but not otherwise, the locus of $a$ includes as part of itself a system of lines; or, say, that it is made up of these lines, and of a residuai curve of the order $\omega+\omega^{\prime}-$ Red., which is the proper locus.

\section{Article Nos. 15 to 17. Application of the foregoing Theory as to the locus of $(a)$.}

15. Reverting now to the case where the angle $a$ is not a free angle but is situate on a given curve $a$, then if the curve $a$ is distinct from the curves $e, F$, the number of positions of $a$ is, as was seen, $g=\chi+\chi^{\prime}$. But the points in question are the intersections of the curve $a$ with the locus of $a$ considered as a free angle; and hence in the case $B=F$, but not otherwise, they are made up of the intersections of the curve $a$ with the system of lines, and of its intersections with the proper locus of $a$. But the intersections with the system of lines are improper solutions of the problem (or, to use a locution which may be convenient, they are "heterotypic" solutions): the true solutions are the intersections with the proper locus of $a$; and the number of these is not $\chi+\chi^{\prime},=a\left(\omega+\omega^{\prime}\right)$, but it is $=a\left(\omega+\omega^{\prime}-\right.$ Red. $)$; say it is $=\chi+\chi^{\prime}-$ Red., where the symbol "Red." is now used to signify a times the number of lines, or reduction in the expression $\omega+\omega^{\prime}-$ Red. of the order of the proper locus of $a$.

16. It is however to be noticed that if the curve $a$, being as is assumed distinct from the curves $e$, and $F=B$, is identical with one or both of the remaining curves $c, D$, the foregoing expression $\chi+\chi^{\prime}-$ Red. may include positions which are not true solutions of the problem, viz. the curve a may pass through special points on the proper locus of $a$, giving intersections which are a new kind of heterotypic solutions $\left({ }^{1}\right)$.

1 More generally, if the curve $a$ be a curve identical with any of the other curves, then if treating in the first instance the angle $a$ as free we find in any manner the locus of $a$, the required positions of the angle $a$ are the intersections of this locus and of the curve $a$; but these intersections will in general include intersections which give heterotypic solutions. The determination of these is a matter of some delicacy, and I have in general treated the problems in such manner that the question does not arise; but as an example see post, Case 43. 
17. But this cannot happen if the curve $a$ is distinct also from the curves $c, D$; or, say, simply when $a$ is a distinct curve. The conclusion is, that in the case where $a$ is a distinct curve we have

$$
\mathrm{g}=\chi+\chi^{\prime}-\mathrm{Red}
$$

where the term "Red." vanishes except in the case of the identity $B=F$ of the curves $B, F$; and that when this identity subsists it is $=a$ times the reduction in the order of the locus of $a$ considered as a free angle; viz. this consists of a firstmode and a second-mode reduction as above explained.

\section{Article Nos. 18 to 21. Remarks in regard to the Solutions for the 52 Cases.}

18. Before going further I remark that the principle of correspondence applies to corresponding and united tangents in like manner as to corresponding and united points, and that all the investigations in regard to the in-and-circumscribed triangle might thus be presented in the reciprocal form, where, instead of points and lines, we have lines and points respectively. But there is no occasion to employ any such reciprocal process; the result to which it would lead is the reciprocal of a result given by the original process, and as such it can always be obtained by reciprocation of the original result, without any performance of the reciprocal process.

19. It is hardly necessary to remark that although reciprocal results would, by the employment of the two processes respectively, be obtained in a precisely similar manner, yet that this is not so when only one of the reciprocal processes is made use of; so that, using one process only, it may be and in general is easier and more convenient to obtain directly one than the other of two reciprocal results; for instance, to consider the case $B=D=F^{\prime}$ rather than $a=c=e$, or vice vers $\hat{a}$; and that it is sufficient to do this, and having obtained the one result, directly to deduce from it the other by reciprocity; but that it may nevertheless be interesting to obtain each of the two results directly.

20. It is moreover obvious that although the several forms of the same case, for instance Case 2, $a=c, a=e$, or $c=e$, are absolutely equivalent to each other, yet that, when as above we select a vertex $a$, and seek for the number of the united points $(a, g)$, the process of obtaining the result will be altogether different according to the different form which we employ. For instance, in the case just referred to, if the form is taken to be $a=c$ or $c=e$, then the equation $\mathrm{g}=\chi+\chi^{\prime}$ is applicable to it; but not so if the form is taken to be $a=e$. It would be by no means uninteresiing in every case to consider the several forms successively and get out the result from each of them; I shall not, however, do this, but only consider two or more forms of the same case when for comparison, illustration, verification, or otherwise it appears proper so to do. The translation of a result, for instance, of a form $a=e$ or $c=e$ into that for the form $a=c=x$ is so easy and obvious, that it is not even necessary formally to make it. 
21. I do not at present further consider the general theory, but proceed to consider in order the 52 cases, interpolating in regard to the general theory such further discussion or explanation as may appear necessary. In the several instances in which the equation $g=\chi+\chi^{\prime}$ is applicable, it is sufficient to write down the values of $\chi, \chi^{\prime}$, the mode of obtaining these being already explained.

The 52 Cases for the in-and-circumscribed triangles.

Case 1. No identities.

$$
\begin{aligned}
& \chi=B c D e F a, \quad \chi^{\prime}=F e D c B a(=\chi) \\
& g=2 a c e B D F .
\end{aligned}
$$

Case 2. $a=c=x$.

$$
\begin{aligned}
& \chi=B(x-1) D e F x, \quad \chi^{\prime}=F e D x B(x-1)(=\chi) \\
& g=2 x(x-1) e B D F .
\end{aligned}
$$

Second process, for form $a=e=x$. The equation of correspondence is here

$$
\mathrm{g}-\chi-\chi^{\prime}+F^{\prime}\left(\mathrm{e}-\epsilon-\epsilon^{\prime}\right)=0
$$

but the points $e$ being given as all the intersections of the curve $a(=e)$ by the linesystem $c D e$ which does not pass through $a$, we have $e-\epsilon-\epsilon^{\prime}=0$; so that $\mathrm{g}=\chi+\chi^{\prime}$; and then

$$
\chi=B c D x F^{\prime}(x-1), \quad \chi^{\prime}=F(x-1) D c B x,
$$

giving the former result( ${ }^{(1)}$.

Case 3. $D=F=x$. Reciprocation from 2 ; or else, second process,

$$
\begin{aligned}
& \chi=B c X e(X-1) a, \quad \chi^{\prime}=X e(X-1) c B a, \\
& g=2 X(X-1) \text { Bace. }
\end{aligned}
$$

Third process: form $F=B=x$. We have here $\mathrm{g}=\chi+\chi^{\prime}-$ Red.

$$
\begin{aligned}
& \chi=X c D e X a, \quad \chi^{\prime}=X_{e} D_{c} X a(=\chi), \\
& \chi+\chi^{\prime}=2 X^{2} \text { Dace }
\end{aligned}
$$

and the reductions are those of the first and second mode, as explained ante, Nos. 11,12 , viz. each of these is $=X$ Dace, and together they are $=2 X D a c e$; whence the foregoing result.

Case 4. $a=D=x$.

$$
\begin{aligned}
& \chi=B c X e F x, \quad \chi^{\prime}=F e X B x(=\chi), \\
& g=2 X x c e B F .
\end{aligned}
$$

1 Of course, the result is obtained in the form belonging to the new form of specification, viz. here it is $=2 x(x-1) c B D F$; and so in other instances; but it is unnecessary to refer to this change. 
Observe this is what the result for Case 1 becomes on writing therein $a=D=x$, viz. the opposite curves $a, D$ may become one and the same curve without any alteration in the form of the result.

Case 5. $a=B=x$.

$$
\chi=(X-2) c D e F x, \quad \chi^{\prime}=F e D c X(x-2),
$$

where

therefore

$$
(X-2) x+X(x-2)=2(X x-X-x)
$$

$$
\mathrm{g}=2(X x-X-x) c e D F .
$$

Case 6. $a=c=e=x$ : perhaps most easily by reciprocation of Case 7 ; or

Second process, functionally by taking the curve $a=c=e$ to be the aggregate curve $x+x^{\prime}$. The triangle $a B c D e F$ is here in succession each of the eight triangles:

$$
\begin{array}{l|l}
x B x D x F & x^{\prime} B x^{\prime} D x^{\prime} F \\
x^{\prime}, x, x, & x, x^{\prime}, x^{\prime}, \\
x, x^{\prime}, x, & x^{\prime}, x, x^{\prime}, \\
x^{\prime}, x, x, & x, x^{\prime}, x^{\prime},
\end{array}
$$

where the two top triangles give $\phi x$ and $\phi x^{\prime}$ respectively; the remaining triangles all belong to Case 2, and those of the first column give each $2\left(x^{2}-x\right) x^{\prime} B D F$, and those of the second column each $2\left(x^{\prime 2}-x^{\prime}\right) x B D F$. We have thus

$$
\phi\left(x+x^{\prime}\right)-\phi x-\phi x^{\prime}=\left\{6\left(x^{2} x^{\prime}+x x^{\prime 2}\right)-12 x x^{\prime}\right\} B D F .
$$

Hence obtaining a particular solution and adding the constants, we have

$$
\phi x=\left(2 x^{3}-6 x^{2}+\alpha x+\beta X+\gamma \xi\right) B D F ;
$$

it is easy to see that $\alpha, \beta, \gamma$ are independent of the curves $B, D, F$; and taking each of these to be a point, and the curve $a=c=e$ to be a conic, then it is known that $\phi x=2$; we have therefore $2=16-24+2 \alpha+2 \beta+6 \gamma$, that is $\alpha+\beta+3 \gamma=5$.

The case where the curve $a=c=e$ is a line gives $0=2-6+\alpha+3 \gamma$, that is $\alpha+3 \gamma=4$; but it is not easy to find another condition; assuming however $\gamma=0$, we have $\alpha=4$, $\beta=1$, and thence

$$
\phi x=\left(2 x^{3}-6 x^{2}+4 x+X\right) B D F,
$$

or say

$$
\mathrm{g}=\{2 x(x-1)(x-2)+X\} B D F:
$$

this is a good easy example of the functional process, the use of which begins to exhibit itself; and I have therefore given it, notwithstanding the difficulty as to the complete determination of the constants. 
Third process. The equation of correspondence is

$$
\mathrm{g}-\chi-\chi^{\prime}+F\left(\mathrm{e}-\epsilon-\epsilon^{\prime}\right)=0
$$

but for the correspondence $(a, e)$ we have

$$
\mathrm{e}-\epsilon-\epsilon^{\prime}+D\left(\mathrm{c}-\gamma-\gamma^{\prime}\right)=0,
$$

and for the correspondence $(a, c)$ we have

whence

$$
\mathrm{c}-\gamma-\gamma^{\prime}=B \Delta \text {, }
$$

and then

$$
\mathrm{g}=\chi+\chi^{\prime}+B D F \cdot \Delta
$$

that is

$$
\chi=B(x-1) D(x-1) F^{\prime}(x-1), \quad \chi^{\prime}=F^{\prime}(x-1) D(x-1) B(x-1)(=\chi) ;
$$

Moreover

$$
\chi+\chi^{\prime}=B D F \cdot 2(x-1)^{3} \text {. }
$$

$$
\Delta=X-2 x+2+\kappa
$$

(if $\kappa$ be the number of cusps of the curve $a=c=e$ ), and the resulting value is

that is

$$
\mathrm{g}=\left\{2(x-1)^{3}+X-2 x+2+\kappa\right\} B D F
$$

$$
=\{2 x(x-1)(x-2)+X+\kappa\} B D F,
$$

where, however, the term $\kappa B D F$ is to be rejected. I cannot quite explain this; I should rather have expected a rejection $=2 \kappa B D F$, introducing the term $-\kappa$. For consider a tangent from the curve $D$ from a cusp of the curve $a=c=e$ : there are $D$ such tangents; each gives in the neighbourhood of the cusp two points, say $c, e$; and from these we draw $B$ tangents $c B a$ to the curve $B$, and $F$ tangents $e F a$ to the curve $F$; we have thus in respect of the given tangent of $D, B F$ positions of $a$, or in all $B D F$ positions of $a$ which will ultimately coincide with the cusp; that is, $B D F$ infinitesimal triangles of which the angles $a, c, e$ coincide together at the cusp; and for all the cusps together $\kappa B D F$ such triangles: this would be what is wanted; the difficulty is that as (of the two intersections at the cusp) each in succession might be taken for $c$, and the other of them for $e$, it would seem that the foregoing number $\kappa B D F$ should be multiplied by 2 .

Case 7. $B=D=F=x$. Here $g=\chi+\chi^{\prime}-$ Red. and

that is,

$$
\chi=X c(X-1) e(X-1) a, \quad \chi^{\prime}=X e(X-1) c(X-1) a(=\chi) \text {; }
$$

$$
\chi+\chi^{\prime}=2 X(X-1)^{2} \text { ace. }
$$

The reductions of the two modes are as above, with only the variation that in the present case $D$ is the same curve with the two curves $B=F$. That of the first mode is $=X(X-1)$ ace, and that of the second mode is $(2 \tau+3 \iota)$ ace, which is $=\{X(X-1)-x\}$ ace; together they are $=\{2 X(X-1)-x\}$ ace, or subtracting, we have

$$
\mathrm{g}=\{2 X(X-1)(X-2)+x\} \text { ace. }
$$


Case 8. $a=c=B=x$.

$$
\begin{aligned}
& \chi=(X-2)(x-3) D e F x, \quad \chi^{\prime}=F e D x(X-2)(x-3)(=\chi), \\
& g=2 x(x-3)(X-2) e D F .
\end{aligned}
$$

Case 9. $D=F=e=x$. By reciprocation of 8 .

$$
\text { No. }=2 X(X-3)(x-2) a c B \text {. }
$$

Case 10. $a=c=D=x$.

$$
\begin{aligned}
& \chi=B(x-1)(X-2) e F x, \quad \chi^{\prime}=F^{\prime} e X(x-2) B(x-1), \\
& g=2(x-1)(X x-X-x) e B F .
\end{aligned}
$$

Case 11. $D=F=a=x$. By reciprocation of 10 .

$$
\text { No. }=2(X-1)(X x-X-x) c e B \text {. }
$$

Second process : form $a=B=D=x$.

$$
\chi=(X-2) c(X-1) e F x, \quad \chi^{\prime}=F e X c(X-1)(x-2),
$$

giving the former result.

Case 12. $c=e=x, a=D=y$.

$$
\begin{aligned}
& \chi=B x Y(x-1) F y, \quad \chi^{\prime}=F x Y(x-1) B y(=\chi) \\
& \mathrm{g}=2 x(x-1) y Y B F .
\end{aligned}
$$

Case 13. $F=B=x, a=D=y$. By reciprocation of 12 .

$$
\text { No. }=2 X(X-1) \text { Yyce. }
$$

Case 14. $c=e=x, a=B=y$.

$$
\begin{aligned}
& \chi=(Y-2) x D(x-1) F y, \quad \chi^{\prime}=F x D(x-1) Y(y-2), \\
& \mathrm{g}=2 x(x-1)(Y y-Y-y) D F
\end{aligned}
$$

Case 15. $F=B=x, D=e=y$. By reciprocation of 14 .

$$
\text { No. }=2 X(X-1)(Y y-Y-y) a c \text {. }
$$

Case 16. $c=e=x, D=F=y$.

$$
\begin{aligned}
& \chi=B x Y(x-1)(Y-1) a, \quad \chi^{\prime}=Y x(Y-1)(x-1) B a(=\chi), \\
& \mathrm{g}=2 x(x-1) Y(Y-1) a B .
\end{aligned}
$$

Case 17. $c=e=x, B=F=y$.

$$
\begin{aligned}
& \chi=D(x-1) Y a(Y-1) x, \quad \chi^{\prime}=Y a(Y-1) x D(x-1)(=\chi) \\
& \mathrm{g}=2 x(x-1) Y(Y-1) a D .
\end{aligned}
$$

But we have here $a D$ as an axis of symmetry, so that each triangle is counted twice, or the number of distinct triangles is $=\frac{1}{2} \mathrm{~g}$. 
Case 18. $a=D=x, c=B=y$.

$$
\begin{aligned}
& \chi=Y(y-2) X e F x, \quad \chi^{\prime}=F e X y(Y-2) x(=\chi), \\
& g=2 x X(Y y-Y-y) e F
\end{aligned}
$$

Case 19. $c=F=x, e=B=y$.

$$
\begin{aligned}
& \chi=Y x D y X a, \quad \chi^{\prime}=X y D x Y a(=\chi), \\
& g=2 x y X Y a D .
\end{aligned}
$$

Case 20. $c=D=x, e=F=y$.

$$
\begin{aligned}
\chi & =B x(X-2) y(Y-2) a, \quad \chi^{\prime}=Y(y-2) X(x-2) B a, \\
g & =\{x y(X-2)(Y-2)+X Y(x-2)(y-2)\} a B \\
& =2\{x y X Y-x y(X+Y)-X Y(x+y)+2 x y+2 X Y\} a B .
\end{aligned}
$$

Case 21. $c=B=x, e=F=y$.

$$
\begin{aligned}
\chi & =X(x-2) D y(Y-2) a, \quad \chi^{\prime}=Y(y-2) D x(X-2) a \\
g & =\{X(Y-2) y(x-2)+Y(X-2) x(y-2)\} a D \\
& =2\{x y X Y-x y(X+Y)-X Y(x+y)+2 x Y+2 y X\} a D .
\end{aligned}
$$

Case 22. $a=D=x, c=F=y, e=B=z$.

$$
\begin{aligned}
& \chi=Z y X z Y x, \quad \chi^{\prime}=Y z X y Z x(=\chi), \\
& g=2 x y z X Y Z
\end{aligned}
$$

Case 23. $a=B=x, c=D=y, e=F=z$.

$$
\begin{aligned}
\chi= & (X-2) y(Y-2) z(Z-2) x, \quad \chi^{\prime}=Z(z-2) Y(y-2) X(x-2), \\
g= & x y z(X-2)(Y-2)(Z-2)+X Y Z(x-2)(y-2)(z-2) \\
= & 2\{x y z X Y Z-x y z(Y Z+Z X+X Y)-X Y Z(y z+z x+x y) \\
& +2 x y z(X+Y+Z)+2 X Y Z(x+y+z)-4 x y z-4 X Y Z\} .
\end{aligned}
$$

Case 24. $a=D=x, c=B=y, e=F=z$.

$$
\begin{aligned}
\chi & =Y(y-2) X z(Z-2) x, \quad \chi^{\prime}=Z(z-2) X y(Y-2) x \\
g & =x X\{Y(Z-2) z(y-2)+Z(Y-2) y(z-2)\} \\
& =2 x X\{y z Y Z-y z(Y+Z)-Y Z(y+z)+2 y Z+2 z Y\} .
\end{aligned}
$$

Case 25. $a=c=x, D=F=y, e=B=z$.

$$
\begin{aligned}
& \chi=Z(x-1) Y z(Y-1) x, \quad \chi^{\prime}=Y z(Y-1) x Z(x-1)(=\chi) \\
& \mathrm{g}=2 x(x-1) Y(Y-1) z Z
\end{aligned}
$$

But we have here $e B$ as an axis of symmetry, so that each triangle is counted twice, or the number of distinct triangles is $=\frac{1}{2} \mathrm{~g}$.

C. VIII. 
Case 26. $a=c=x, B=D=y, e=F=z$.

$$
\begin{aligned}
\chi & =Y(x-1)(Y-1) z(Z-2) x, \quad \chi^{\prime}=Z(z-2) Y x(Y-1)(x-1) \\
\mathrm{g} & =x(x-1) Y(Y-1)\{z(Z-2)+Z(z-2)\} \\
& =2 x(x-1) Y(Y-1)(z Z-z-Z) .
\end{aligned}
$$

Case 27. $a=c=e=x, B=F=y$. By reciprocation of 28 .

$$
\text { No. }=\{2 x(x-1)(x-2)+X\} Y(Y-1) D,
$$

where each triangle is counted twice, so that the number is really one half of this.

Case 28. $B=D=F=x, c=e=y$.

Here

$$
\begin{aligned}
& \mathrm{g}=\chi+\chi^{\prime}-\text { Red. } \\
& \chi=X y(X-1)(y-1)(X-1) a, \quad \chi^{\prime}=X y(X-1)(y-1)(X-1) a(=\chi) \\
& \chi+\chi^{\prime}=a y(y-1) .2 X(X-1)^{2} .
\end{aligned}
$$

The reductions are those of the first and second mode as explained above, with the variation that the curves $c$ and $e$ are here identical, $c=e$, and that the curve $D$ is identical with the curves $B=F$.

First-mode reduction is

$$
a(C+2 \delta+3 \kappa) B(B-1)
$$

(where $\delta, \kappa$ refer to the curve $c=e$ ), which is

$$
=a c(c-1) B(B-1) \text {; }
$$

that is, the reduction is $=a y(y-1) X(X-1)$.

Second-mode reduction is

$$
a(2 \tau+3 \iota) c(c-1)
$$

(where $\tau, \iota$ refer to the curve $B=D=F^{\prime}$ ), which is

$$
=a\{B(B-1)-b\} c(c-1) ;
$$

that is, the reduction is $=a y(y-1)\{X(X-1)-x\}$.

Hence the two together are $=a y(y-1)\{2 X(X-1)-x\}$; and subtracting from $\chi+\chi^{\prime}$ we have

$$
\mathrm{g}=a y(y-1) \cdot\{2 X(X-1)(X-2)+x\}
$$

but on account of the symmetry each triangle is reckoned twice, and the number of triangles is $=\frac{1}{2} \mathrm{~g}$.

Case 29. $a=c=B=x, D=F=y$.

$$
\begin{aligned}
& \chi=(X-2)(x-3) Y e(Y-1) x, \quad \chi^{\prime}=Y e(Y-1) x(X-2)(X-3)(=\chi), \\
& \mathrm{g}=2 x(x-3)(X-2) Y(Y-1) e .
\end{aligned}
$$


Second process. Taking the form

here

$$
C=D=e=x, \quad B=F=y
$$

and

$$
\begin{aligned}
\text { No. } & =\chi+\chi^{\prime}-\text { Red. } \\
\chi & =Y x(X-2)(x-3) Y a,=\gamma^{\prime},
\end{aligned}
$$

$$
\chi+\chi^{\prime}=2 Y^{2} x(x-3)(X-2) a .
$$

There is a first-mode reduction,

viz. this is

$$
a Y\{2 \tau+2 \delta(X-4)+3 \kappa(X-3)\}
$$

which is

$$
\begin{aligned}
a Y\{ & X^{2}-X+8 x-3 \xi \\
+ & (X-4)\left(x^{2}-x+8 X-3 \xi\right) \\
+ & (X-3)(\quad-9 X+3 \xi)\}
\end{aligned}
$$

$$
=a Y\left\{X\left(x^{2}-x-6\right)-4 x^{2}+12 x\right\} ;
$$

and a second-mode reduction

$$
=a Y X(x-2)(x-3) .
$$

Hence the two together are

whence the result is

$$
\begin{aligned}
& =a Y\left\{X\left(2 x^{2}-6 x\right)-4 x^{2}+12 x\right\} \\
& =2 Y x(x-3)(X-2) a,
\end{aligned}
$$

$$
=2\left(Y^{2}-Y\right) x(x-3)(X-2) a,
$$

which agrees with that obtained above.

On account of the symmetry we must divide by 2 .

Case 30. $e=D=F=x, a=c=y$. By reciprocation of 29 .

$$
\text { No. }=2 X(X-3)(x-2) y(y-1) B .
$$

On account of the symmetry we must divide by 2 .

Case 31. $c=e=D=x, a=B=y$.

$$
\begin{aligned}
\chi & =(Y-2) x(X-2)(x-3) F y, \quad \chi^{\prime}=F x(X-2)(x-3) Y(y-2), \\
\mathrm{g} & =x(x-3)(X-2) F\{(Y-2) y+Y(y-2)\} . \\
& =2 x(x-3)(X-2)(y Y-y-Y) F .
\end{aligned}
$$

Case 32. $F=B=a=x, D=e=y$. By reciprocation of 31 .

$$
\text { No. }=2 X(X-3)(x-2)(y Y-y-Y) c .
$$


Case 33. $B=F=y, a=e=D=x$. By reciprocation of 34 .

$$
\text { No. }=2(x-1)(x X-x-X) Y(Y-1) c .
$$

Case 34. $c=e=y, B=D=a=x$.

$$
\begin{aligned}
\chi & =(X-2) y(X-1)(y-1) F x, \quad \chi^{\prime}=F y X(y-1)(X-1)(x-2), \\
g & =y(y-1)(X-1)\{(X-2) x+X(X-2)\} F \\
& =2(X-1)(x X-x-X) y(y-1) F .
\end{aligned}
$$

Case 35. $a=D=y, c=e=B=x$.

$$
\begin{aligned}
\chi & =X(x-2) Y(x-1) F y, \quad \chi^{\prime}=F x Y(x-1)(X-2) y \\
g & =y Y(x-1)\{X(x-2)+(X-2) x\} F \\
& =2(x-1)(x X-x-X) y Y F
\end{aligned}
$$

Case 36. $a=D=y, B=F=e=x$. By reciprocation of 35 .

$$
\text { No. }=2(X-1)(X x-x-X) y Y c .
$$

Case 37. $a=e=D=x, c=B=y$. By reciprocation of 38 .

$$
\text { No. }=2(x-1)\{x y X Y-x y(X+Y)-X Y(x+y)+2 x y+2 X Y\} F \text {. }
$$

Case 38. $B=D=a=x, F=e=y$.

$$
\begin{aligned}
\chi & =(X-2) c(X-1) y(Y-2) x, \quad \chi^{\prime}=Y(y-2) X c(X-1)(x-2), \\
g & =(X-1) c\{x y(X-2)(Y-2)+X Y(x-2)(y-2)\} \\
& =2(X-1)\{x y X Y-x y(X+Y)-X Y(x+y)+2 x y+2 X Y\} c .
\end{aligned}
$$

Case 39. $a=c=e=B=x$.

Functional process; the curve is assumed to be the aggregate of two curves, say $a=c=e=B=x+x^{\prime}$. Forming the enumeration

\begin{tabular}{l|cr}
$x X x D x F$ & Case \\
$x^{\prime} X x \cdot x$. & $x^{\prime} X^{\prime} x^{\prime} D x^{\prime} F$ & 39 \\
$x X^{\prime} x \cdot x$. & $\& c$. & 10 \\
$x^{\prime} X^{\prime} x . x$. &. & 6 \\
$x X x^{\prime} . x$. &. & 14 \\
$x^{\prime} X x^{\prime} \cdot x$. &. & 10 \\
$x X^{\prime} x^{\prime} \cdot x$. &. & 12 \\
$x^{\prime} X^{\prime} x^{\prime} \cdot x$. &. & 14 \\
&. & 8
\end{tabular}

(where the second column is derived from the first by a mere interchange of the accented and unaccented letters), I annex to each line the number of the case to 
which it belongs; thus $x^{\prime} X x D x F$ is $B=c=e=x$, which is Case 10, and so in the other instances. Observing that cases 10 and 14 occur each twice, we have thus

$$
\begin{aligned}
& \phi\left(x+x^{\prime}\right)-\phi x-\phi x^{\prime}=D F \text { multiplied into } \\
& \\
& 4(x-1)(X x-X-x) x^{\prime}+\ldots(10) \times 2 \\
&+\{2 x(x-1)(x-2)+X\} X^{\prime}+\ldots \\
&+ 4 x(x-1)\left(X^{\prime} x^{\prime}-X^{\prime}-x^{\prime}\right)+\ldots(14) \times 2 \\
&+ 2 x(x-1) x^{\prime} X^{\prime} \\
&+ 2 x(x-3)(X-2) x^{\prime} \\
&
\end{aligned}
$$

where the (..)'s refer to the like functions with the two sets of letters interchanged. Developing and collecting, this is

$$
\begin{aligned}
\phi\left(x+x^{\prime}\right)- & \phi x-\phi x^{\prime}=D F \text { multiplied into } \\
& 2 X X^{\prime} \\
+ & 2 X\left(3 x^{2} x^{\prime}+3 x x^{\prime 2}+x^{\prime 3}-10 x x^{\prime}-5 x^{\prime 2}+6 x^{\prime}\right) \\
+ & 2 X^{\prime}\left(x^{3}+3 x^{2} x^{\prime}+3 x x^{\prime 2}-5 x^{2}-10 x x^{\prime}+6 x\right) \\
- & 12\left(x^{2} x^{\prime}+x x^{\prime 2}\right)+40 x x^{\prime},
\end{aligned}
$$

and thence

$$
\begin{array}{cc}
\phi x= & D F \text { multiplied into } \\
& X^{2} \\
+ & X\left(2 x^{3}-10 x^{2}+12 x\right)-L X \\
- & 4 x^{3}+20 x^{2}
\end{array}
$$

where the constants $L, l, \lambda$ have to be determined. Now for a cubic curve the number of triangles vanishes; that is, we have $\phi x=0$ in each of the three cases,

$$
\begin{array}{ccc}
x=3, & X=6, & \xi=18, \\
& X=4, & \xi=12, \\
& X=3, & \xi=10
\end{array}
$$

and we thus obtain the three equations

$$
\begin{aligned}
& 0=108-6 L-3 l-18 \lambda, \\
& 0=88-4 L-3 l-12 \lambda, \\
& 0=81-3 L-3 l-10 \lambda,
\end{aligned}
$$

giving $L=1, l=16, \lambda=3$. Whence, finally,

$$
\phi x=\left\{X^{2}+X\left(2 x^{3}-10 x^{2}+12 x-1\right)-4 x^{3}+20 x^{2}-16 x-3 \xi\right\} D F .
$$

Second process, by correspondence. We have

$$
\begin{aligned}
& \mathrm{g}-\chi-\chi^{\prime}+F\left(\mathrm{e}-\epsilon-\epsilon^{\prime}\right)=0 \\
& \mathrm{e}-\epsilon-\epsilon^{\prime}+D\left(\mathrm{c}-\gamma-\gamma^{\prime}\right)=0
\end{aligned}
$$


and thence

Moreover

$$
\mathrm{g}-\chi-\chi^{\prime}=D F\left(\mathrm{c}-\gamma-\gamma^{\prime}\right)
$$

and

$$
\begin{aligned}
\chi & =(X-2)(x-3) D(x-1) F^{\prime}(x-1), \\
\chi^{\prime} & =F^{\prime}(x-1) D(x-1)(X-2)(x-1),=\chi, \\
\chi+\chi^{\prime} & =D F(X-2) 2(x-3)(x-1)^{2},
\end{aligned}
$$

$$
c-\gamma-\gamma^{\prime}=2 \tau+(X-3) \kappa-2(X-2)(x-3),
$$

as is easily obtained, but see also post, No. 29; hence

$$
\begin{aligned}
g=D F & \text { multiplied into } \\
& (X-2) \cdot 2(x-3)(x-1)^{2} \\
+ & (X-2) \cdot-2(x-3) \\
+ & 2 \tau+(X-3) \kappa
\end{aligned}
$$

but I reject the term $D F \cdot(X-3) \kappa$ as in fact giving a heterotypic solution; I do not go into the explanation of this. And then substituting for $2 \tau$ its value, we have

$$
\begin{aligned}
& \mathrm{g}=D F \text { multiplied into } \\
& (X-2) \cdot 2 x(x-1)(x-2) \\
& +X^{2}-X+8 x-3 \xi \text {, }
\end{aligned}
$$

where the second factor is

$$
=X^{2}+X\left(2 x^{3}-10 x^{2}+12 x-1\right)-4 x^{3}+20 x^{2}-16 x-3 \xi,
$$

which is the foregoing result.

Case 40. $B=D=F^{\prime}=e=x$. By reciprocation of 39 .

$$
\text { No. }=\left\{x^{2}+x\left(2 X^{3}-10 X^{2}+12 X-1\right)-4 X^{3}+20 X^{2}-16 X-3 \xi\right\} \text { ac. }
$$

Case 41. $c=e=D=F=x$.

$$
\begin{aligned}
\chi & =B x(X-2)(x-3)(X-3) a \\
\chi^{\prime} & =X(x-2)(X-3)(x-3) B a \\
g & =(x-3)(X-3) a B\{x(X-2)+X(x-2)\} \\
& =2 \quad(x-3)(X-3)(x X-x-X) a B .
\end{aligned}
$$

Case 42. $a=c=D=F=x$.

Functional Process; the curve is supposed to be the aggregate of two curves, say $a=c=D=F=x+x^{\prime}$. 
The enumeration is

whence

$$
\begin{array}{l|c}
x B x X e X & x^{\prime} B x^{\prime} X^{\prime} e X^{\prime}, \\
x^{\prime} \cdot x X \cdot X & \text { \&c. } \\
x \cdot x^{\prime} X \cdot X & \\
x^{\prime} \cdot x^{\prime} X \cdot X & \\
x \cdot x X^{\prime} \cdot X & \\
x^{\prime} \cdot x X^{\prime} \cdot X & \\
x \cdot x^{\prime} X^{\prime} \cdot X & \\
x^{\prime} \cdot x^{\prime} X^{\prime} \cdot X &
\end{array}
$$

$$
\begin{aligned}
& \phi\left(x+x^{\prime}\right)-\phi x-\phi x^{\prime}=e B \text { multiplied into } \\
& 4(X-1)(X x-X-x) x^{\prime} \\
& +\ldots(11) \times 2 \\
& +2 x(x-1) X^{\prime}\left(X^{\prime}-1\right) \\
& +\ldots(17) \\
& +4(x-1)(X x-x-x) X^{\prime} \\
& +\ldots(10) \times 2 \\
& +2 x X x^{\prime} X^{\prime} \\
& +\ldots(19) \\
& +2 x x^{\prime} X X^{\prime}-2\left(x+x^{\prime}\right) X X^{\prime}-2\left(X+X^{\prime}\right) x x^{\prime}+4\left(X x^{\prime}+X^{\prime} x\right)+\ldots
\end{aligned}
$$

where the (..)'s refer to the like functions with the two sets of letters interchanged. Developing and collecting, we have

$$
\begin{aligned}
\phi\left(x+x^{\prime}\right)-\phi x-\phi x^{\prime}=e B & \text { multiplied into } \\
& X^{2} \quad\left(4 x x^{\prime}+2 x^{\prime 2}-6 x^{\prime}\right) \\
& +X X^{\prime}\left(4 x^{2}+8 x x^{\prime}+4 x^{\prime 2}-12 x-12 x^{\prime}+8\right) \\
& +X^{\prime 2} \quad\left(2 x^{2}+4 x x^{\prime}-6 x\right) \\
+ & \left(-12 x x^{\prime}-6 x^{\prime 2}+18 x^{\prime}\right) \\
+X^{\prime} & \left(-6 x^{2}-12 x x^{\prime}+18 x\right) \\
+ & 8 x x^{\prime}
\end{aligned}
$$

and consequently

$$
\begin{array}{lc}
\phi x= & e B \text { multiplied into } \\
& X^{2}\left(2 x^{2}-6 x+4\right) \\
+ & X\left(-6 x^{2}+18 x+L\right) \\
+ & 4 x^{2}+l x+\lambda \xi
\end{array}
$$

where the constants $L, l, \lambda$ have to be determined. The number of triangles vanishes when the curve is a line or a conic, that is $\phi x=0$ for $x=1, X=0, \xi=0$, and for $x=X=2, \xi=6$; we thus have

$$
\begin{aligned}
& 0=4+l, \\
& 0=40+2 L+2 l+6 \lambda .
\end{aligned}
$$


Moreover, the data being sibireciprocal, the result must be so likewise; we must therefore have $L=l$. We thus obtain $L=l=\lambda=-4$; so that finally

$$
\phi x=\left\{X^{2}\left(2 x^{2}-6 x+4\right)+X\left(-6 x^{2}+18 x-4\right)+4 x^{2}-4 x-4 \xi\right\} e B .
$$

Second process, by correspondence: form $a=c=D=F=x$. We have

$$
c-\chi-\chi^{\prime}+2\left(\mathrm{f}-\phi-\phi^{\prime}\right)=0
$$

also from the special consideration that the points $D, F$ are given as the intersections of the curve $x$, by the first polar of the point $e$, which first polar does not pass through $a$, we have

$$
\left(\mathrm{f}-\phi-\phi^{\prime}\right)+e\left(\mathrm{~d}-\delta-\delta^{\prime}\right)=0
$$

and by the consideration that $c, D$ are given as intersections, $c$ a double intersection, of the curve with the first polar of the point $c$, which first polar does not pass through $a$,

whence

$$
\mathrm{d}-\delta-\delta^{\prime}+2\left(\mathrm{c}-\gamma-\gamma^{\prime}\right)=0,
$$

$$
\mathrm{g}-\chi-\chi^{\prime}=-4 e\left(\mathrm{c}-\gamma-\gamma^{\prime}\right)
$$

and

so that this is

$$
c-\gamma-\gamma^{\prime}=B \Delta
$$

$$
\begin{aligned}
g-\chi-\chi^{\prime} & =-4 B e \Delta \\
& =-4 B e(-2 X-2 x+2+\xi)
\end{aligned}
$$

Also

$$
\begin{aligned}
& \chi=B(x-1)(X-2) e(X-1)(x-2), \\
& \chi^{\prime}=(X-2) e(X-1)(x-2) B(x-1),=\chi
\end{aligned}
$$

so that

$$
\mathrm{g}=B e \text { multiplied into }
$$

$$
2(X-1)(X-2)(x-1)(x-2)-4(-2 X-2 x+2+\xi),
$$

viz. this is

$$
B e\left\{X^{2}\left(2 x^{2}-6 x+4\right)+X\left(-6 x^{2}+18 x-4\right)+4 x^{2}-4 x-4 \xi\right\}
$$

Third process: form $c=e=F=B=x$.

$$
\begin{aligned}
\mathrm{g} & =\chi+\chi^{\prime}-\text { Red., } \\
\chi & =X(x-2) D(x-1)(X-2) a, \\
\chi^{\prime} & =X(x-2) D(x-1)(X-2) a,=\chi, \\
\chi+\chi^{\prime} & =a D \cdot 2 X(X-2)(x-1)(x-2) .
\end{aligned}
$$

The first-mode reduction is here

$$
a D[(X-2) X+(X-4) 2 \delta+(X-3) 3 \kappa+\kappa] ;
$$


where the last term $a D \kappa$ arises from the tangents $c B a$ and $e F a$, each coinciding with a cuspidal tangent, as shown in the figure.

Fig. 4.

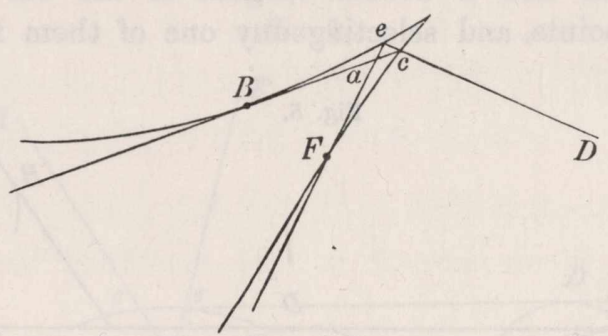

The second-mode reduction is

$$
=a D \cdot X(x-2)(x-3),
$$

so that the two reductions together are

viz. this is

$$
=a D\{(X-2) X+(X-4) 2 \delta+(X-3) 3 \kappa+\kappa+X(x-2)(x-3)\}
$$

$$
=a D\{(X-2) X+(X-4)(2 \delta+3 \kappa)+4 \kappa+X(x-2)(x-3)\} ;
$$

or substituting for $2 \delta+3 \kappa$ and $\kappa$ the values $x^{2}-x-X$ and $-3 X+\xi$ respectively, and reducing, it is

$$
a D\left\{X\left(2 x^{2}-6 x-4\right)-4 x^{2}+4 x+4 \xi\right\} .
$$

Hence subtracting from $\chi+\chi^{\prime}$, written in the form

the result is

$$
a D\left\{X^{2}\left(2 x^{2}-6 x+4\right)+X\left(-4 x^{2}+12 x-8\right)\right\},
$$

$$
=a D\left\{X^{2}\left(2 x^{2}-6 x+4\right)+X\left(-6 x^{2}+18 x-4\right)+4 x^{2}-4 x-4 \xi\right\} .
$$

On account of the symmetry we must divide by 2 .

Case 43. $a=c=e=x, B=D=F=y$.

Suppose for a moment that the angle $a$ is a free point; the locus of $a$ is a curve the order of which is obtained from Case 28, by writing $c=e=x, B=D=F=y$; the locus in question meets a curve order $a$ in $\{2 Y(Y-1)(Y-2)+y\} x(x-1) a$ points; wherefore the order of the locus is

$$
=\{2 Y(Y-1)(Y-2)+y\} x(x-1),
$$

and this locus meets the curve $a=c=e=x$ in a number of points

$$
=\{2 Y(Y-1)(Y-2)+y\} x^{2}(x-1) \text {, }
$$

viz. this is the number of positions of the angle $a$; but several of these belong to special forms of the triangle $a B c D e F$, giving heterotypic solutions, which are to be rejected; the required number is thus

$$
\{2 Y(Y-1)(Y-2)+y\} x^{2}(x-1)-\text { Red. }
$$

c. VIII. 
The reduction is due first and secondly to triangles wherein the angle $a$ coincides with an angle $c$ or $e$, and thirdly to triangles wherein the angles $a, c, e$ all coincide.

$1^{\circ}$. Take for the side $c D e$ a double tangent of the curve $B=D=F$, this meets the curve $a=c=e$ in $x$ points, and selecting any one of them for $e$ and any other for $c$,

Fig. 5 .

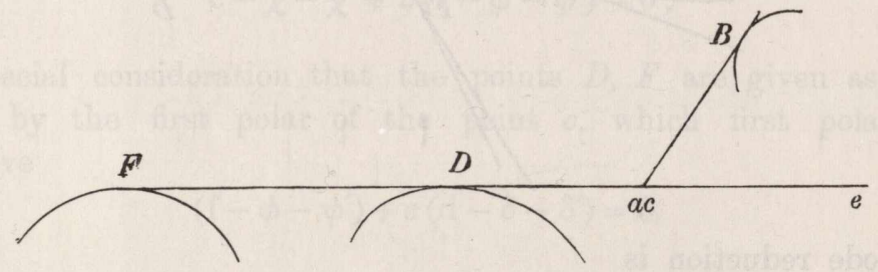

we have from the last-mentioned point $Y-2$ tangents to the curve $B=D=F$; and in respect of each of these a position of $a$ coincident with $c$. The reduction on this account is $2 \tau x(x-1)(Y-2)$; but since we may in the figure interchange $c$ and $e$, $B$ and $F$, we have the same number belonging to the coincidence of the angles $a, e$, or together the reduction is $=4 \tau x(x-1)(Y-2)$.

Fig. 6 .

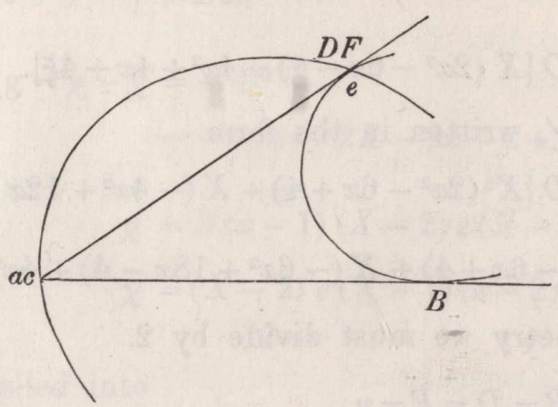

But instead of a double tangent we may have $c D e$ a stationary tangent; we have thus reductions $3 \iota x(x-1)(Y-2)$ and $3 \iota x(x-1)(Y-2)$, together $6 \iota x(x-1)(Y-2)$; and for the double and stationary tangents together we have

that is

$$
\begin{aligned}
& (4 \tau+6 \imath) x(x-1)(Y-2), \\
= & 2\{Y(Y-1)-y\} x(x-1)(Y-2), \\
= & 2 x(x-1) Y(Y-1)(Y-2)-2 x(x-1) y(Y-2) .
\end{aligned}
$$

$2^{\circ}$. The side $c D e$ may be taken to be a tangent to the curve $B=D=F$ at any one of its intersections with the curve $a=c=e$. Taking then the point $e$ at the intersection in question, and the point $c$ at any other of the intersections of the tangent with the curve $a=c=e$, and from $c$ drawing any other tangent to the curve $B=D=F$, there is in respect of each of these tangents a position of $a$ at $c$; and 
the reduction on this account is $=x y(x-1)(Y-1)$. But interchanging in the figure the letters $c, e, B, F$, there is an equal reduction belonging to the coincidence of $a, e$; and the whole reduction in this manner is $=2 x(x-1) y(Y-1)$.

Fig. 7.

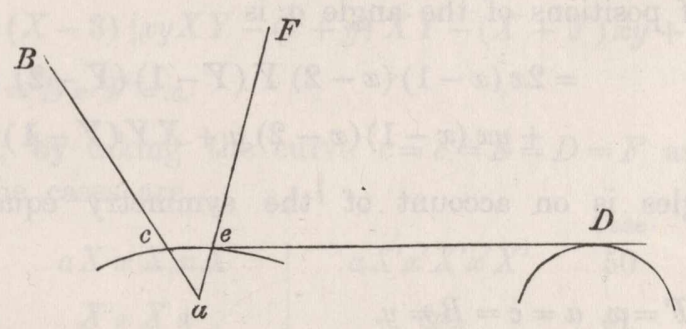

$3^{\circ}$. If the side $c D e$ intersects the curve $a=c=e$ in two coincident points, then taking these in either order for the points $c, e$, and from the two points respectively drawing two other tangents to the curve $D=B=F$, we have a triangle wherein the angles $a, c, e$ all coincide. The side $c D e$ may be a proper tangent to the curve $a=c=e$, or it may pass through a node or a cusp of this curve, viz. it is either a common tangent of the curves $B=D=F$ and $a=c=e$ (as in the figure, except that for greater distinctness the points $c$ and $e$ are there drawn nearly instead of actually coincident), or it may be a tangent to the curve $B=D=F$ from a node or a cusp of the curve $a=c=e$; we have thus the numbers

$\begin{array}{ll}\text { Common tangent } & X Y(Y-1)(Y-2), \\ \text { Tangent from node } & 2 \delta Y(Y-1)(Y-2), \\ \text { Tangent from cusp } & 2 \kappa Y(Y-1)(Y-2) ;\end{array}$

but (as we are counting intersections with the curve $a=c=e$ ) the second of these, as being at a node of this curve, is to be taken 2 times; and the third, as being at a cusp, 3 times; and the three together are thus

$$
\begin{aligned}
& (X+4 \delta+6 \kappa) Y(Y-1)(Y-2), \\
= & \{2 x(x-1)-X\} Y(Y-1)(Y-2) .
\end{aligned}
$$

The reductions $1^{\circ}, 2^{\circ}, 3^{\circ}$ altogether are

$$
\begin{aligned}
& 2 x(x-1) Y(Y-1)(Y-2) \\
& -2 x(x-1) y(Y-2) \\
& +2 x(x-1) y(Y-1) \\
& +2 x(x-1) Y(Y-1)(Y-2) \\
& -X Y(Y-1)(Y-2) \\
& =4 x(x-1) Y(Y-1)(Y-2) \\
& \quad+2 x(x-1) y \\
& -X Y(Y-1)(Y-2)
\end{aligned}
$$

which is 
and subtracting from the before-mentioned number

$$
\begin{aligned}
& 2 x^{2}(x-1) Y(Y-1)(Y-2) \\
& +x^{2}(x-1) y,
\end{aligned}
$$

the required number of positions of the angle $a$ is

$$
\begin{aligned}
= & 2 x(x-1)(x-2) Y(Y-1)(Y-2) \\
& +y x(x-1)(x-2) y+X Y(Y-1)(Y-2) .
\end{aligned}
$$

The number of triangles is on account of the symmetry equal to one-sixth of this number.

Case 44. $e=D=F=x, a=c=B=y$.

$$
\begin{aligned}
& \chi=(Y-2)(y-3) X(x-2)(X-3) y, \\
& \chi^{\prime}=X(x-2)(X-3) y(Y-2)(y-3)(=\chi), \\
& \mathrm{g}=2(x-2) X(X-3)(Y-2) y(y-3):
\end{aligned}
$$

there is a division by 2 on account of the symmetry.

Case 45. $a=D=B=x, c=e=F=y$.

$$
\begin{aligned}
\chi & =(X-2) y(X-1)(y-1)(Y-2) x, \\
\chi^{\prime} & =Y(y-2) X(y-1)(X-1)(x-2), \\
\mathrm{g} & =(X-1)(y-1)\{x y(X-2)(Y-2)+X Y(x-2)(y-2)\} \\
& =2(X-1)(y-1)\{X Y x y-X Y(x+y)-x y(X+Y)+2 x y+2 X Y\} .
\end{aligned}
$$

Case 46. $a=c=y, B=D=F=e=x$. By reciprocation of 47,

$$
\text { No. }=y(y-1)\left\{x^{2}+x\left(2 X^{3}-10 X^{2}+12 X-1\right)-4 X^{3}+20 X^{2}-16 X-3 \xi\right\}:
$$

there is a division by 2 on account of the symmetry.

Case 47. $D=F=y, a=c=e=B=x$.

The functional process is exactly the same as for No. $39(a=c=e=B=x)$, with only $Y(Y-1)$ written instead of $D F$; hence

$$
\text { No. }=Y(Y-1)\left\{X^{2}+X\left(2 x^{3}-10 x^{2}+12 x-1\right)-4 x^{3}+20 x^{2}-16 x-3 \xi\right\}:
$$

there is a division by 2 on account of the symmetry.

Case 48. $a=c=D=F=x, e=B=y$.

The functional process, writing $a=c=D=F=x+x^{\prime}$, would be precisely the same as for Case 42 , with only the factor $y Y$ written instead of $e B$; and we have thus the like result, viz.

$$
\text { No. }=\left\{X^{2}\left(2 x^{2}-6 x+4\right)+X\left(-6 x^{2}+18 x-4\right)+4 x^{2}-4 x-4 \xi\right) y Y,
$$

which on account of the symmetry must be divided by 2 . 
Case 49. $a=B=y, c=e=D=F=x$.

$$
\begin{aligned}
\chi & =(Y-2) x(X-2)(x-3)(X-3) y, \\
\chi^{\prime} & =X(x-2)(X-3)(x-3) Y(y-2), \\
\mathrm{g} & =(x-3)(X-3)\{x y(X-2)(Y-2)+X Y(x-2)(y-2)\} \\
& =2(x-3)(X-3)\{x y X Y-(x+y) X Y-(X+Y) x y+2 x y+2 X Y\} .
\end{aligned}
$$

\begin{tabular}{|c|c|}
\hline$a X x X x X$ & $a X^{\prime} x^{\prime} X^{\prime} x^{\prime} X^{\prime}$ \\
\hline$X^{\prime} x X x$ & $\& c$. \\
\hline$X x^{\prime} X x$ & . \\
\hline$X^{\prime} x^{\prime} X x$ & . \\
\hline$X x X^{\prime} x$ & . \\
\hline$X^{\prime} x X^{\prime} x$ & . \\
\hline$X x^{\prime} X^{\prime} x$ & . \\
\hline$X^{\prime} x^{\prime} X^{\prime} x$ & . \\
\hline$X x X x^{\prime}$ & . \\
\hline$X^{\prime} x X x^{\prime}$ & r \\
\hline$X x^{\prime} X x^{\prime}$ & . \\
\hline$X^{\prime} x^{\prime} X x^{\prime}$ & . \\
\hline$X x X^{\prime} x^{\prime}$ & . \\
\hline$X^{\prime} x X^{\prime} x^{\prime}$ & . \\
\hline$X x^{\prime} X^{\prime} x^{\prime}$ & . \\
\hline$X^{\prime} x^{\prime} X^{\prime} x^{\prime} X$ & . \\
\hline
\end{tabular}

Case 50. $c=e=B=D=F=x$.

Functional process; by taking the curve $c=e=B=D=F$ as the aggregate of two curves, say $=x+x^{\prime}$. The cases are

and we thus have

$$
\begin{aligned}
& \phi\left(x+x^{\prime}\right)-\phi x-\phi x^{\prime}=a \text { multiplied into } \\
& =4(x-3)(X-3)(x X-x-X) X^{\prime}+. . \\
& +2 x^{\prime}\left[x^{2}+x\left(2 X^{3}-10 X^{2}+12 X-1\right)-4 X^{3}+20 X^{2}-16 X-3 \xi\right]+\ldots 2(40) \\
& +4 X(X-3)(x-2)\left(x^{\prime} X^{\prime}-X^{\prime}-x^{\prime}\right)+\ldots 2(32) \\
& +\left[X^{2}\left(2 x^{2}-6 x+4\right)+X\left(-6 x^{2}+18 x-4\right)+4 x^{2}-4 x-4 \xi\right] X^{\prime}+\ldots \quad(42) \\
& +4(x-1)(x X-x-X)\left(X^{\prime 2}-X^{\prime}\right)+\ldots 2(33) \\
& +4(X-1)\left[X X^{\prime} x x^{\prime}-X X^{\prime}\left(x+x^{\prime}\right)-x x^{\prime}\left(X+X^{\prime}\right)+2 X X^{\prime}+2 x x^{\prime}\right]+\ldots 2(38) \\
& +4 x X\left(X^{\prime}-1\right)\left(X^{\prime} x^{\prime}-X^{\prime}-x^{\prime}\right) \\
& +\left(x^{2}-x\right)\left(2 X^{\prime 3}-6 X^{\prime 2}+4 X^{\prime}+x^{\prime}\right) \\
& +.2(36) \\
& +2 x(x-3)(X-2)\left(X^{\prime 2}-X^{\prime}\right) \\
& +\ldots(28) \\
& +\ldots
\end{aligned}
$$


where as before the (. .)'s refer to the like functions with the two sets of letters interchanged. Developing and collecting, this is

$$
\begin{aligned}
& \phi\left(x+x^{\prime}\right)-\phi x-\phi x^{\prime}=a \text { multiplied into } \\
& 3 x^{2} x^{\prime}+3 x x^{\prime 2} \\
&+x^{2} \cdot 6 X^{2} X^{\prime}+6 X X^{\prime 2}+2 X^{\prime 3} \\
&- 28 X X^{\prime}-14 X^{\prime 2} \\
&+ 28 X^{\prime} \\
&+x x^{\prime} .4 X^{3}+12 X^{2} X^{\prime}+12 X X^{\prime 2}+4 X^{\prime 3} \\
&-28 X^{2}-56 X X^{\prime}-28 X^{\prime 2} \\
&+ 56 X+56 X^{\prime} \\
&-22 \\
&+x^{\prime 2}-2 X^{3}+6 X^{2} X^{\prime}+6 X X^{\prime 2} \\
&-14 X^{2}-28 X X^{\prime} \\
&+ 28 X \\
&+x-30 X^{2} X^{\prime}-30 X X^{\prime 2}-10 X^{\prime 3} \\
&+ 140 X X^{\prime}+70 X^{\prime 2} \\
&-116 X^{\prime}-6 \xi \\
&+x^{\prime} .-10 X^{3}-30 X^{2} X^{\prime}-30 X X^{\prime 2} \\
&+ 70 X^{2}+140 X X^{\prime} \\
&-116 X-6 \xi \\
&+ 36 X^{2} X^{\prime}+36 X X^{\prime 2} \\
&-152 X X^{\prime} \\
&- 4\left(X \xi^{\prime}+X^{\prime} \xi\right)
\end{aligned}
$$

whence

$$
\begin{aligned}
\phi x= & a \text { multiplied into } \\
& x^{3}(\quad+1) \\
& +x^{2}\left(2 X^{3}-14 X^{2}+28 X-11\right) \\
& +x\left(-10 X^{3}+70 X^{2}-116 X+l\right) \\
& +\quad 12 X^{3}-76 X^{2} \quad+L X \\
& +\xi(-6 x-4 X \quad+\lambda),
\end{aligned}
$$

where the constants $l, L, \lambda$ have to be determined. We should have $\phi x=0$ for a cubic curve; viz. $x=3: X=6, \xi=18 ; X=4, \xi=12$; or $X=3, \xi=10$. Writing first $x=3$, the equation is

$$
8 X^{2}-96 X-72-\xi(18+4 X)+3 l+X L+\xi \lambda=0,
$$


giving in the three cases respectively

$$
\begin{aligned}
& 3 l+6 L+18 \lambda=1116 \\
& 3 l+4 L+12 \lambda=736 \\
& 3 l+3 L+10 \lambda=588
\end{aligned}
$$

and we have then $l=-8, L=64, \lambda=42$, so that the required number is

$$
\begin{aligned}
& =x^{3}(+1) \\
& +x^{2}\left(2 X^{3}-14 X^{2}+28 X-11\right) \\
& +x\left(-10 X^{3}+70 X^{2}-116 X-8\right) \\
& +12 X^{3}-76 X^{2}+64 X \\
& +\xi(-6 x-4 X+42) \text {. }
\end{aligned}
$$

As a verification, observe that for a conic, $x=X=2, \xi=6$, this is $=0$.

Second process, by correspondence: form $c=e=B=D=F=x$.

We have

$$
\begin{aligned}
& g=\chi+\chi^{\prime}-\text { Red., } \\
& \chi=X(x-2)(X-3)(x-3)(X-3) a \\
& \chi^{\prime}=X(x-2)(X-3)(x-3)(X-3) a,=\chi \\
& \chi+\chi^{\prime}=a \text { into } \\
& 2(x-2)(x-3) X(X-3)^{2}
\end{aligned}
$$

Fig. 8.

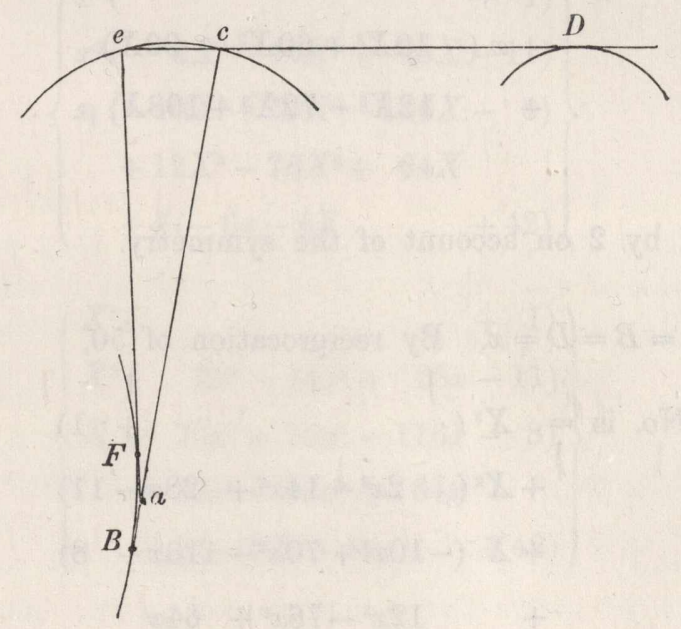

There is a first-mode reduction, which is

$$
=a\{2 \delta(X-4)(X-5)+3 \kappa(X-3)(X-4)+\kappa(X-3)+2 \tau(X-3)\} \text {, }
$$


where the term $a .2 \tau(X-3)$ arises, as shown in the figure, and a second-mode reduction, which is

$$
=a\{2 \tau(x-4)(x-5)+3 \iota(x-3)(x-4)\}
$$

and the two together are $=a$ into

$$
\begin{array}{rrr} 
& (X-4)(X-5)\left(x^{2}-x+8 X\right. & -3 \xi) \\
+ & (X-3)(X-4)\left(\begin{array}{rr} 
\\
+
\end{array}\right. \\
+ & (X-3)\left(\begin{array}{rr}
-3 X & +3 \xi) \\
+X^{2}-X+8 x-3 \xi
\end{array}\right) \\
+ & (x-4)(x-5)\left(X^{2}-X+8 x\right. & -3 \xi) \\
+ & (x-3)(x-4)( & -9 x+3 \xi)
\end{array}
$$

that is, $=a$ into

$$
\begin{aligned}
& -x^{3} \\
& +x^{2} \cdot 2 X^{2}-10 X+11 \\
& +x \cdot-10 X^{2}+26 X+8 \\
& +4 X^{2}+44 X \\
& +\xi(6 x+14 X-4 x)
\end{aligned}
$$

and subtracting this from the foregoing value of $\chi+\chi^{\prime}$, which is $=a$ into

$$
\begin{aligned}
& x^{2}\left(2 X^{3}-12 X^{2}+18 X\right) \\
& +x\left(-10 X^{3}+60 X^{2}-90 X\right) \\
& +\quad 12 X^{3}-72 X^{2}+108 X \text {, }
\end{aligned}
$$

the result is as before.

There is a division by 2 on account of the symmetry.

Case 51. $a=c=e=B=D=x$. By reciprocation of 50 ,

$$
\begin{aligned}
\text { No. is }= & X^{3}(\quad+1) \\
& +X^{2}\left(2 x^{3}-14 x^{2}+28 x-11\right) \\
& +X\left(-10 x^{3}+70 x^{2}-116 x-8\right) \\
& +\quad 12 x^{3}-76 x^{2}+64 x \\
& +\xi(-6 X-4 x+42) .
\end{aligned}
$$

There is a division by 2 on account of the symmetry. 
Case 52. $a=c=e=B=D=F=x$.

Functional process, by taking the curve to be the aggregate of two curves, say $=x+x^{\prime}$. The enumeration of the cases is conveniently made in a somewhat different manner from that heretofore employed, viz. we may write

\begin{tabular}{c|ccc}
$\begin{array}{c}x \text { or } x^{\prime} \\
\|\end{array}$ & $x^{\prime}$ or $x$ & Case & times \\
all & $\|$ & & \\
$a$ & none & $(52)$ & 1 \\
$B$ & residue & $(50)$ & 3 \\
$a, c$ & $\#$ & $(51)$ & 3 \\
$B, D$ & $"$ & $(46)$ & 3 \\
$a, D$ & $"$ & $(47)$ & 3 \\
$a, B$ & $"$ & $(48)$ & 3 \\
$a, c, e$ & " & $(49)$ & 6 \\
$a, B, F$ & $c, e, D$ & $(43)$ & 1 \\
$a, B, D$ & $c, e, F$ & $(44)$ & 3 \\
& & & \\
& & &
\end{tabular}

and the functional equation then is

$$
\left.\begin{array}{c}
\phi\left(x+x^{\prime}\right)-\phi x-\phi x^{\prime} \\
=3 x^{\prime} \\
\left.+3 X^{\prime} \quad\left\{\begin{array}{lr}
x^{3}( & +1) \\
x^{2} & \left.2 X^{3}-14 X^{2}+28 X-11\right) \\
x\left(-10 X^{3}+70 X^{2}-116 X-8\right) \\
+12 X^{3}-76 X^{2}+64 X \\
+\xi(-6 x-4 X
\end{array}\right\}+.42\right)
\end{array}\right\}
$$

C. VIII. 


$$
\begin{aligned}
& +3\left(X^{\prime 2}-X^{\prime}\right)\left\{\begin{array}{l}
X^{2} \\
+X\left(2 x^{3}-10 x^{2}+12 x-1\right) \\
-4 x^{3}+20 x^{2}-16 x-3 \xi
\end{array}\right\}+\ldots \\
& +3 x^{\prime} X^{\prime} \quad\left\{\begin{array}{r}
X^{2}\left(2 x^{2}-6 x+4\right) \\
+X\left(-6 x^{2}+18 x-4\right) \\
+4 x^{2}-4 x-4 \xi
\end{array}\right\} \\
& +\ldots \quad(48) \times 3 \\
& +12\left(x^{\prime}-3\right)\left(X^{\prime}-3\right)\left\{x x^{\prime} X X^{\prime}-x x^{\prime}\left(X+X^{\prime}\right)-X X^{\prime}\left(x+x^{\prime}\right)+2 x x^{\prime}+2 X X^{\prime}\right\}+\ldots(49) \times 6 \\
& +\left\{2 x^{\prime}\left(x^{\prime}-1\right)\left(x^{\prime}-2\right) X(X-1)(X-2)+x x^{\prime}\left(x^{\prime}-1\right)\left(x^{\prime}-2\right)+X^{\prime} X(X-1)(X-2)\right\}+\ldots \\
& +6\left(x^{\prime}-2\right) X^{\prime}\left(X^{\prime}-3\right)(X-2)(x-3) \quad+\ldots(44) \times 3 \\
& +12\left(X^{\prime}-1\right)(x-1)\left\{x x^{\prime} X X^{\prime}-x x^{\prime}\left(X+X^{\prime}\right)-X X^{\prime}\left(x+x^{\prime}\right)+2 x x^{\prime}+2 X X^{\prime}\right\}+\ldots(45) \times 6
\end{aligned}
$$

where as before the (..)'s refer to the like functions with the two sets of letters interchanged. Developing and collecting, this is found to be

$$
\begin{gathered}
4 X^{3} X^{\prime}+6 X^{2} X^{\prime 2}+4 X X^{\prime 3} \\
+X^{3}\left\{\begin{array}{c}
6 x^{2} x^{\prime}+6 x x^{\prime 2}+2 x^{\prime 3} \\
-36 x x^{\prime}-18 x^{\prime 2} \\
+52 x^{\prime}
\end{array}\right\} \\
+\left(X^{2} X^{\prime}+X X^{\prime 2}\right) \\
+X^{\prime 3}\left\{\begin{array}{c}
6 x^{3}+18 x^{2} x^{\prime}+18 x x^{\prime 2}+6 x^{\prime 3} \\
-54 x^{2}-108 x x^{\prime}-54 x^{\prime 2} \\
+156 x+156 x^{\prime} \\
-138 \\
-18 x^{2}-6 x^{2} x^{\prime}+6 x x^{\prime 2} \\
+52 x
\end{array}\right\}
\end{gathered}
$$$$
+\& c \text {. \&c. }
$$

I abstain from writing down the remaining terms, as they can at once be obtained backwards from the value of $\phi x$; they were in fact found directly, and the integration of the functional equation then gives

$$
\begin{aligned}
& \phi x= \\
& X^{4}(+1) \\
& +X^{3}\left(\quad 2 x^{3}-18 x^{2}+52 x-46\right) \\
& +X^{2}\left(-18 x^{3}+162 x^{2}-420 x+221\right) \\
& +X\left(52 x^{3}-420 x^{2}+704 x+l\right) \\
& +\quad x^{4}-46 x^{3}+221 x^{2}+l x \\
& +\xi\left\{\begin{array}{c}
X^{2}(-9) \\
+X(-12 x+135) \\
-9 x^{2}+135 x+\lambda
\end{array}\right\}
\end{aligned}
$$


where the constants $l, \lambda$ have to be determined; I have in the first instance written $l(X+x)+\lambda \xi$, instead of $L X+l x+\lambda \xi$, thus introducing two constants only, since it is clear from the symmetry in regard to $x, X$ that we must have $l=L$. We must have $\phi x=0$, when the curve is a conic or cubic. Writing $x=2$, we have

$$
\phi x=X^{4}+2 X^{3}-115 X^{2}+144 X+532+\xi\left(-9 X^{2}+111 X+234\right)+l(2+X)+\xi \lambda,
$$

and then for the conic, $X=2, \xi=6$.

Writing $x=3$, we have

$$
\phi x=X^{4}+2 X^{3}-67 X^{2}-264 X+828+\xi\left(-9 X^{2}+99 X+324\right)+l(3+X)+\lambda \xi,
$$

and then for the three cases of the cubic $X=6, \xi=18 ; X=4, \xi=12$; and $X=3, \xi=10$. We have thus the four equations

$$
\begin{aligned}
& 2912+4 l+6 \lambda=0 \\
& 9252+9 l+18 \lambda=0 \\
& 5796+7 l+12 \lambda=0 \\
& 4968+6 l+10 \lambda=0,
\end{aligned}
$$

all satisfied by $l=+172, \lambda=-600$. Hence finally

$$
\begin{aligned}
& \phi x=\quad X^{4}(+1) \\
& +X^{3}\left(2 x^{3}-18 x^{2}+52 x-46\right) \\
& +X^{2}\left(-18 x^{3}+162 x^{2}-420 x+221\right) \\
& +X\left(\quad 52 x^{3}-420 x^{2}+704 x+172\right) \\
& +\quad x^{4}-46 x^{3}+221 x^{2}+172 x \\
& +\xi\left\{\begin{array}{cr}
X^{2} & \left(\begin{array}{r}
9 \\
X
\end{array}\right. \\
+X & -12 x+135) \\
-9 x^{2}+135 x-600
\end{array}\right\}
\end{aligned}
$$

but on account of the symmetry the number of triangles is $=$ one-sixth of this expression.

Article Nos. 22 to 36. The Case 52, as belonging to a different series of Problems.

22. In the foregoing Case 52, where all the curves are one and the same curve, we have the unclosed trilateral $a B c D e F g$, and we seek for the number of the united points $(a, g)$. But we may consider this as belonging to a series of questions, viz. we may seek for the number of the united points $(a, B),(a, c),(a, D),(a, e),\left(a, F^{\prime}\right)$, (the last four of these giving by reciprocity the numbers of the united points $(B, D),(B, e)$, $(B, F),(B, g))$, and finally the number of the united points $(a, g)$. It is very instructive to consider this series of questions, and the more so that in those which precede $(a, F)$ there are only special solutions having reference to the singular points and tangents of the curve, and that the solutions thus explain themselves. 
23. Thus the first case is that of the united points $(a, B)$, viz. we have here a point $a$ on the curve, and from it we draw to the curve a tangent $a B$ touching it at $B$; the points $a$ and $B$ are to coincide together. Observe that from a point in general $a$ of the curve we have $X-2$ tangents ( $X$ the class as heretofore), viz. we disregard altogether the tangent at the point, counting as 2 of the $X$ tangents from a point not on the curve, and attend exclusively to the $X-2$ tangents from the point. Now if the point $a$ is an inflection, or if it is a cusp, there are only $X-3$ tangents, or, to speak more accurately, one of the $X-2$ tangents has come to coincide with the tangent at the point; such tangent is a tangent of three-pointic intersection, viz. we have the point $a$ and the point $B$ (counting, as a point of contact, twice) all three coinciding; that is, we have a position of the united point $(a, B)$; and the number of these united points is $=\iota+\kappa$.

24. It is important to notice that neither a point of contact of a double tangent, nor a double point, is a united point. In the case of the point of contact of a double tangent, one of the tangents from the point coincides with the double tangent; but the point $B$ is here the other point of contact of this tangent, so that the points $a, B$ are not coincident. In the case of a double point, regarding the assumed position of $a$ at the double point as belonging to one of the two branches, then of the $X-2$ tangents there are two, each coinciding with the tangent to the other branch; hence, attending to either of these, the point $B$ belongs to the other branch, and thus, though $a$ and $B$ are each of them at the double point, the two do not constitute a united point. (In illustration remark that for a unicursal curve, the position of $a$ answers to a value $=\lambda$, and that of $B$ to a value $=\mu$ of the parameter $\theta$, viz. $\lambda, \mu$ are the two values of $\theta$ at the double point; contrariwise in the foregoing case of a cusp, where there is a single value $\lambda=\mu$. Hence the whole number of the united points $(a, B)$ is $=\iota+\kappa$, and this is in fact the value given, as will presently appear, by the theory of correspondence.)

I recall that I use $\Delta,=2 D$, to denote twice the deficiency of the curve, viz. that we have $\Delta=X-2 x+2+\kappa,=-2 x-2 X+2+\xi$.

25. The several cases are

United points.
$(a, B) \quad \mathrm{b}-\beta-\beta^{\prime}=2 \Delta$,
$(a, c) \quad c-\gamma-\gamma^{\prime}+2\left(b-\beta-\beta^{\prime}\right)=(X-2) \Delta$,
$(B, D) \quad \mathrm{c}_{0}-\gamma_{0}-\gamma_{0}^{\prime}$ by reciprocity,
$(a, D) \quad \mathrm{d}-\delta-\delta^{\prime}+2\left(\mathrm{c}_{0}-\gamma_{0}-\gamma_{0}^{\prime}\right)+(X-3)\left(\mathrm{b}-\beta-\beta^{\prime}\right)=0$,
$(a, e) \quad$ e $-\epsilon-\epsilon^{\prime}+2\left(\mathrm{~d}-\delta-\delta^{\prime}\right)+(X-3)\left(\mathrm{c}-\gamma-\gamma^{\prime}\right)=0$,
$\left(B, F^{\prime}\right) \quad \mathrm{e}_{0}-\epsilon_{0}-\epsilon_{0}^{\prime}$ by reciprocity,
$\left(a, F^{\prime}\right) \quad \mathrm{f}-\phi-\phi^{\prime}+2\left(\mathrm{e}_{0}-\epsilon_{0}-\epsilon_{0}^{\prime}\right)+(X-3)\left(\mathrm{d}-\delta-\delta^{\prime}\right)=0$,
$(a, g) \quad \mathrm{g}-\chi-\chi^{\prime}+2\left(\mathrm{f}-\phi-\phi^{\prime}\right)+(X-3)\left(\mathrm{e}-\epsilon-\epsilon^{\prime}\right)=0$,
$(B, H) \quad g_{0}-\chi_{0}-\chi_{0}^{\prime}$ by reciprocity,

and so on. 
26. The mode of obtaining these equations appears ante, Nos. 5 and 6 , but for greater clearness I will explain it in regard to a pair of the equations, say those for $(a, e),(a, D)$. Regarding $a$ as given, we draw from $a$ the tangents $a B c$, touching at $B$ and besides intersecting at $c$ (viz. the number of tangents is $=X-2$, and the number of the points $c$ is $=(X-2)(x-3))$; from each of the positions of $c$ we draw to the curve the $(X-3)$ tangents $c D e$ touching at $D$ and intersecting at $e$; the whole number of these tangents is $=(X-2)(x-3)(X-3)$; and this is also the number of the points $D$, but the number of the points $e$ is $=(X-2)(x-3)(X-3)(x-3)$. Now this system of the $(X-2)(x-3)(X-3)$ tangents is the curve $\Theta$ of the general theory (ante, Nos. 3, 4), viz. the curve $\Theta$ (which does not pass through a) intersects the given curve in the three classes of points $c, D, e$, the number of intersections at a point $e$ being $=1$, at a point $D$ being $=2$, and at a point $c$ being $=X-3$. And we have thus the equation

$$
e-\epsilon-\epsilon^{\prime}+2\left(d-\delta-\delta^{\prime}\right)+(X-3)\left(c-\gamma-\gamma^{\prime}\right)=0,
$$

where e, d, c are the numbers of united points and $\left(\epsilon, \epsilon^{\prime}\right),\left(\delta, \delta^{\prime}\right),\left(\gamma, \gamma^{\prime}\right)$ the correspondences in the three cases respectively.

27. Observe that we cannot, starting from $a$, obtain in this manner the equation for the number of the united points $(a, D)$; for we introduce per force the points $e$, and thus obtain the foregoing equation for $(a, e)$. But starting from $D$, the tangent at this point besides intersects the curve in $(x-2)$ points, each of which is a position of $c$; and from each of these drawing a tangent $c B a$ to the curve, we have the curve $\Theta$ consisting of these $(x-2)(X-3)$ tangents, not passing through $D$, but intersecting the given curve in the three classes of points $c, B$, $a$, viz. the number of intersections at each point $c$ is $=X-3$, at each point $B$ it is $=2$, and at each point $a$ it is $=1$; and we have thus the equation

$$
\left(d-\delta-\delta^{\prime}\right)+2\left(c_{0}-\gamma_{0}-\gamma_{0}{ }^{\prime}\right)+(X-3)\left(b-\beta-\beta^{\prime}\right)=0,
$$

where the numbers $\left(\mathrm{d}, \delta, \delta^{\prime}\right),\left(\mathrm{c}_{0}, \gamma_{0}, \gamma_{0}^{\prime}\right),\left(\mathrm{b}, \beta, \beta^{\prime}\right)$ refer to the correspondences $(D, a)$, $(D, B)$, and $(D, c)$ (or what is the same thing $(a, B))$ respectively.

28. Correspondence $(a, B)$.

We have

and thence

$$
\beta=X-2, \quad \beta^{\prime}=x-2,
$$

$$
\begin{aligned}
\mathrm{b} & =x+X-4+2 \Delta \\
& =-3 x-3 X+2 \xi,
\end{aligned}
$$

which is the solution: the value obtained above was $b=\iota+\kappa$, and we in fact have identically

$$
\iota+\kappa=-3 x-3 X+2 \xi .
$$

It was in this manner that I originally applied the principle of correspondence to investigating the number of inflections of a curve, regarding, however, the term $\kappa$ as a special solution; it is better to put the cusp and inflection on the same footing as above. 
29. Correspondence $(a, c)$.

Since $b-\beta-\beta^{\prime}=2 \Delta$, we have here

$$
c-\gamma-\gamma^{\prime}=(X-6) \Delta,
$$

and

whence

$$
\gamma=\gamma^{\prime}=(X-2)(x-3),
$$

$$
\begin{aligned}
c & =2(X-2)(x-3)+(X-6)(-2 x-2 X+2+\xi) \\
& =-2 X^{2}+8 X+8 x+(X-6) \xi
\end{aligned}
$$

this is in fact $=2 \tau+(X-3) \kappa$, viz. we have

$$
\begin{aligned}
2 \tau & =X^{2}-X+8 x-3 \xi \\
(X-3) \kappa & =(X-3)(-3 X+\xi)=-3 X^{2}+9 X+(X-3) \xi
\end{aligned}
$$

and therefore

$$
2 \tau+(X-3) \kappa=\text { as above, }
$$

viz. the united points $(a, c)$ are the $2 \tau$ points of contact of the double tangents, and the $\kappa$ cusps each $(X-3)$ times in respect of the $(X-3)$ tangents from it to the curve. This is the way in which I originally applied the principle to finding the number of double tangents of a curve.

30. Correspondence $(B, D)$. By reciprocation

$$
\begin{aligned}
c_{0}-\gamma_{0}-\gamma_{0}^{\prime} & =(x-6) \Delta, \\
c_{0} & =-2 x^{2}+8 x+8 X+(x-6) \xi \\
& =2 \delta+(x-3) \iota .
\end{aligned}
$$

31. It may be remarked, as regards the cases which follow, that although the result in terms of $(\delta, \kappa, \iota, \tau)$ when once known can be explained and verified easily enough, there is great risk of oversight if we endeavour to find it in the first instance; while on the other hand the transformation from the form in terms of $(x, X, \xi)$, as given by the principle of correspondence, to the required form in terms of $(\delta, \kappa, \iota, \tau)$ is by no means easy. I in fact first obtained the expression in $(x, X, \xi)$, and then, knowing in some measure the form of the other expression, was able to find it by the actual transformation of the expression in $(x, X, \xi)$.

32. Correspondence $(a, D)$.

From the values of $c_{0}-\gamma_{0}-\gamma_{0}^{\prime}$ and $b-\beta-\beta^{\prime}$ we have

and then

$$
\begin{aligned}
\mathrm{d}-\delta-\delta^{\prime} & =-(2 X+2 x-18) \Delta, \\
\delta & =(X-2)(x-3)(X-3), \quad \delta^{\prime}=(x-2)(X-3)(x-3), \\
\mathrm{d} & =(x-3)(X-3)(X+x-4) \\
& \quad+(-2 X-2 x+18)(-2 X-2 x+2+\xi)
\end{aligned}
$$


which is

$$
\begin{aligned}
& =X^{2}(\quad x+1) \\
& +X\left(x^{2}-2 x-19\right) \\
& +\quad x^{2}-19 x \\
& +\xi(-2 X-2 x+18)
\end{aligned}
$$

And then, by means of the equations

we verify that

$$
\begin{aligned}
& (x-4) 2 \tau=(x-4)\left(X^{2}-X+8 x-3 \xi\right), \\
& (X-4) 2 \delta=(X-4)\left(x^{2}-x+8 X-3 \xi\right), \\
& (x-3) \iota=(x-3)(-3 x+\xi), \\
& (X-3) \kappa=(X-3)(-3 X+\xi),
\end{aligned}
$$

$$
\mathrm{d}=(x-4) 2 \tau+(X-4) 2 \delta+(x-3) \iota+(X-3) \kappa .
$$

33. Correspondence $(a, e)$.

From the values of $d-\delta-\delta^{\prime}, c-\gamma-\gamma^{\prime}$ we have

and then

$$
\mathrm{e}-\epsilon-\epsilon^{\prime}=\left(-X^{2}+13 X+4 x-54\right) \Delta \text {, }
$$

that is

$$
\epsilon=\epsilon^{\prime}=(X-2)(x-3)(X-3)(x-3) ;
$$

$$
\begin{aligned}
e=2 & (x-3)^{2}(X-2)(X-3) \\
& +\left(-X^{2}+13 X+4 x-54\right)(-2 X-2 x+2+\xi)
\end{aligned}
$$

which is

and then

$$
\begin{aligned}
& =X^{3}(2) \\
& +X^{2}\left(2 x^{2}-10 x-10\right) \\
& +X\left(-10 x^{2}+26 x+44\right) \\
& +\quad 4 x^{2}+44 x \\
& +\xi\left(-X^{2}+13 X+4 x-54\right) \text {, }
\end{aligned}
$$

$$
\begin{aligned}
(x-4)(x-5) 2 \tau & =(x-4)(x-5)\left(X^{2}-X+8 x-3 \xi\right) \\
\{(X-4)(X-5)+x-3\} 2 \delta & =\{(X-4)(X-5)+x-3\}\left(x^{2}-x+8 X-3 \xi\right), \\
\{3(x-3)(x-4)+x-3\} \iota & =(x-3)(3 x-11)(-3 x+\xi) \\
2(X-3)(X-4) \kappa & =2(X-3)(X-4)(-3 X+\xi)
\end{aligned}
$$

and summing these values and comparing,

$$
\begin{aligned}
\mathrm{c}= & (x-4)(x-5) 2 \tau+2(X-3)(X-4) \kappa \\
& +[(X-4)(X-5)+x-3] 2 \delta+[3(x-3)(x-4)+x-3] \iota .
\end{aligned}
$$

The united points $(a, e)$ are in fact, $1^{\circ}$, each of the $x-4$ intersections of a double tangent with the curve, in respect of the two contacts and of the remaining $x-5$ intersections; $2^{\circ}$, each double point in respect of the two branches and of the pairs of tangents from it to the curve; $3^{\circ}$, each of the $x-3$ intersections of each of the 
tangents at a double point with the curve; $4^{\circ}$, each of the $x-3$ intersections of a tangent at an inflection (stationary tangent) with the curve, in respect of the $(x-4)$ remaining intersections; $5^{\circ}$, each inflection in respect of the $x-3$ intersections of the

Fig. 9.

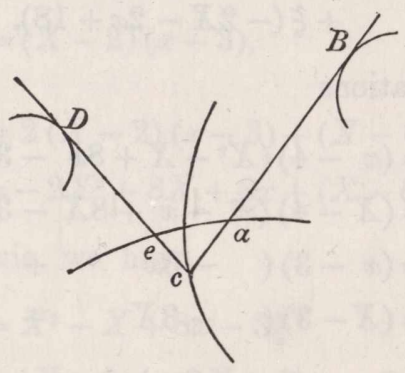

tangent with the curve; and $6^{\circ}$, each cusp in respect of the pairs of tangents from it to the curve. Thus $\left(2^{\circ}\right)$, the double point in respect of the branch which contains $c$, and of the two tangents from it to the curve, is a position of the united point $(a, e)$, as appearing in the figure.

34. Correspondence $\left(B, F^{\prime}\right)$. By reciprocation of $(a, e)$

$$
\begin{aligned}
\mathrm{e}_{0}-\epsilon_{0}-\epsilon_{0}^{\prime}= & \left(-x^{2}+13 x+4 X-54\right) \Delta, \\
\mathrm{e}_{0}= & (X-4)(X-5) 2 \oint+2(x-3)(x-4) \iota \\
& +[(x-4)(x-5)+X-3] 2 \tau+[3(X-3)(X-4)+(X-3)] \kappa .
\end{aligned}
$$

35. Correspondence $\left(a, F^{\prime}\right)$. By means of the values of $\mathrm{e}_{0}-\epsilon-\epsilon^{\prime}$ and $\mathrm{d}-\delta-\delta^{\prime}$, we have

$$
f-\phi-\phi^{\prime}=\left(2 X^{2}+2 X x+2 x^{2}-32 X-32 x+162\right) \Delta,
$$

and then

$$
\begin{aligned}
& \phi=(X-2)(x-3)(X-3)(x-3)(X-3), \\
& \phi^{\prime}=(x-2)(X-3)(x-3)(X-3)(x-3),
\end{aligned}
$$

whence

$$
\begin{aligned}
f= & (X+x-4)(x-3)^{2}(X-3)^{2} \\
& +\left(2 X^{2}+2 X x+2 x^{2}-32 X-32 x+162\right)(-2 X-2 x+2+\xi)
\end{aligned}
$$

which is

$$
\begin{aligned}
& =X^{3}\left(\quad x^{2}-6 x+5\right) \\
& +X^{2}\left(x^{3}-16 x^{2}+61 x-22\right) \\
& +X\left(-6 x^{3}+61 x^{2}-120 x-91\right) \\
& +\quad 5 x^{3}-22 x^{2}-91 x
\end{aligned}
$$

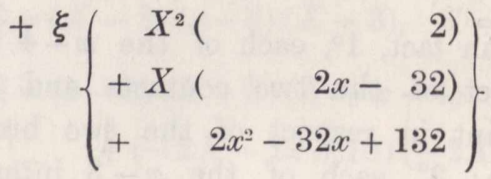


This result includes proper solutions of the problem of finding the number of the triangles $a B c D e F$, which are such that the side $e a$ touches the curve at $a$; and also heterotypic solutions having reference to the singular points of the curve; but I have not determined the number of solutions of each kind.

36. Correspondence $(a, g)$ : from the values of $\mathrm{f}-\phi-\phi^{\prime}$ and $\mathrm{e}-\epsilon-\epsilon^{\prime}$, we have

and then

$$
g-\chi-\chi^{\prime}=\left(X^{3}-20 X^{2}-8 X x-4 x^{2}+125 X+44 x-486\right) \Delta,
$$

wherefore

$$
\chi=\chi^{\prime}=(X-2)(x-3)(X-3)(x-3)(X-\Im)(x-3),
$$

viz. this is

$$
\begin{aligned}
\mathrm{g}= & 2(X-2)(X-3)^{2}(x-3)^{8} \\
& +\left(X^{3}-20 X^{2}-8 X x+125 X+44 x-486\right)(-2 X-2 x+2+\xi),
\end{aligned}
$$

$$
\begin{aligned}
& \mathrm{g}=\tilde{X^{4}}(-2) \\
& +X^{3}\left(2 x^{3}-18 x^{2}+52 x-12\right) \\
& +X^{2}\left(-16 x^{3}+144 x^{2}-376 x+142\right) \\
& +X\left(42 x^{3}-362 x^{2}+780 x+88\right) \\
& -36 x^{3}+236 x^{2}+88 x \\
& +\xi\left\{\begin{array}{rr}
X^{3}( & 1) \\
+X^{2} & -20) \\
+X( & -8 x+125) \\
& +44 x-486
\end{array}\right\}
\end{aligned}
$$

Comparing with the expression of $\phi x$, Case 52 , we have

$$
\begin{aligned}
& \mathrm{g}-\phi x=X^{4}(-3) \\
& +X^{3}(+34) \\
& +X^{2}\left(2 x^{3}-18 x^{2}+44 x-79\right) \\
& +X\left(-10 x^{3}+58 x^{2}+76 x-84\right) \\
& -x^{4}+10 x^{3}+15 x^{2}-84 x \\
& +\xi\left\{\begin{array}{cr}
X^{3}( & 1) \\
+X^{2}( & -11) \\
+X( & 4 x-10) \\
& +9 x^{2}-91 x+114
\end{array}\right\}
\end{aligned}
$$

which difference must be the number of heterotypic solutions having relation to the singularities of the curve; but I have not further considered this.

C. VIII. 